\title{
Three-dimensional simulation of ligament formation and breakup caused by external vibration
}

Cite as: Phys. Fluids 32, 083605 (2020); https://doi.org/10.1063/5.0006817

Submitted: 05 March 2020 . Accepted: 30 July 2020 . Published Online: 12 August 2020

(iD Yikai Li (黎一锴), (D) Mei Zhang (张妹), and (D) Kun Wu (吴坤)

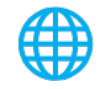

\section{ARTICLES YOU MAY BE INTERESTED IN}

Visualizing the effectiveness of face masks in obstructing respiratory jets

Physics of Fluids 32, 061708 (2020); https://doi.org/10.1063/5.0016018

Head-on collision of two immiscible droplets of different components

Physics of Fluids 32, 082106 (2020); https://doi.org/10.1063/5.0018391

Numerical modeling of the distribution of virus carrying saliva droplets during sneeze and cough

Physics of Fluids 32, 083305 (2020); https://doi.org/10.1063/5.0018432

\section{Physics of Fluids GALLERY OF GOVERS}




\title{
Three-dimensional simulation of ligament formation and breakup caused by external vibration
}

\author{
Cite as: Phys. Fluids 32, 083605 (2020); doi: 10.1063/5.0006817 \\ Submitted: 5 March 2020 - Accepted: 30 July 2020 • \\ Published Online: 12 August 2020
}

Yikai Li (黎一锴), ' (D) Mei Zhang (张妹), ' (D) and Kun Wu (吴坤) ${ }^{2, a)}$ (D)

\begin{abstract}
AFFILIATIONS
${ }^{1}$ School of Mechanical Engineering, Beijing Institute of Technology, Beijing 100081, China

${ }^{2}$ State Key Laboratory of High Temperature Gas Dynamics, Institute of Mechanics, Chinese Academy of Sciences, Beijing 100190, China
\end{abstract}

a) Author to whom correspondence should be addressed: wukun@imech.ac.cn

\begin{abstract}
Ligament formation followed by breakup is the primary process that controls external vibration-driven liquid atomization. In this paper, single-mode Faraday instabilities with detailed interfacial dynamics are studied via three-dimensional simulations with a validated numerical methodology. The detailed mechanisms of ligament formation and its breakup are illuminated. Colliding flow from adjacent troughs results in a pressure increase at the root of the crest. This nonlinear flow structure produces a local maximum pressure point that liberates the liquid region above it from the bulk liquid layer that synchronously moves with the bottom substrate. The appearance of the maximum pressure point can thus be recognized as the indicator of ligament formation. The freed ligament with capillary waves on its surface continues to grow until successive breakup occurs at its tip, which is driven by the "short-wave mode" breakup mechanism. It is found that the tip contraction dynamics of Faraday-type ligament can be well described by a one-dimensional theoretical model of a low-speed liquid jet under temporally periodic acceleration. Finally, the development behaviors of Faraday-type ligament and liquid jet are compared quantitatively, which reveals the analogy in their breakup dynamics in the tip regions.
\end{abstract}

Published under license by AIP Publishing. https://doi.org/10.1063/5.0006817

\section{INTRODUCTION}

Atomization is an efficient way to discretize continuous bulk liquid into much smaller, dispersed droplets. It is commonly used and plays key roles in various scientific and industrial fields. For example, the quality of liquid fuel atomization directly affects spray combustion characteristics in internal combustion engines and gas turbines. ${ }^{2-4}$ Generally, small droplets are expected to meet industrial application requirements because they possess large surface-to-volume ratios and thus are easy to evaporate and mix with surrounding gases. As a result, many techniques have been proposed to promote atomization quality and produce fine sprays.

Liquid atomization is a result of interaction and competition between various forces, such as inertial, aerodynamic, surface tension, and viscous forces. The surface tension acting on the liquid/gas interface and the liquid viscosity usually have stabilizing effects that must be overwhelmed by external, destabilizing forces to achieve liquid atomization. In most combustion-driven power engines, the liquid fuel is injected into the combustion chamber through a highpressure nozzle in order to produce intense turbulence within the fuel jet and a large relative velocity between the fuel jet and surrounding gas, which are favorable to destabilize the interface. ${ }^{5-}$ Thus, high injection pressures are always pursued in modern diesel engines to reduce soot emissions, which increase substantially if the atomized fuel droplets are too large to evaporate quickly. ${ }^{9-1}$ Mechanical vibration can easily provide destabilizing inertial forces and is commonly employed to atomize liquid layers and droplets. The surface acoustic waves that propagate along a substrate surface have been employed to destabilize the air-water interfaces of droplets that sit on such surfaces. ${ }^{12-14}$ An elongated jet forms along the Rayleigh-angle direction when the Weber number $(W e)$ is above 
a critical value. A water mist that consists of multiple child droplets is generated if the $W e$ is increased further. A liquid layer or droplet can burst when it is placed on a plate that is subject to vertical vibration. ${ }^{15-17}$ The characteristics associated with atomization under various vibration conditions and with various liquid properties have been studied systematically. ${ }^{18-22}$ In this type of atomization technology, the sizes of child droplets are inversely proportional to the vibration frequency. In particular, ultrasonic atomization occurs when the vibration frequency exceeds $20 \mathrm{kHz}$. Under these conditions, the typical diameters of atomized droplets are on the order of a micrometer. ${ }^{23-26}$

All atomization can be divided into two sub-processes. First, the ligament, which is typically defined as a large surface deformation extruded from the liquid surface, is formed from the bulk liquid. Next, breakup occurs, and droplets evolve from the ligament. The latter sub-process has attracted extensive academic attention since it directly determines the final atomization characteristics. Most previous studies attribute droplet evolution from a slender filament or jet to the so-called "pinch-off" or "end-pinch" mechanism, which is controlled by complex interactions between inertial, viscous, and capillary forces. ${ }^{6,27-29}$ The We and Ohnesorge numbers and the initial filament aspect ratio greatly affect breakup dynamics. ${ }^{30}$ In addition to the pinch-off mechanism, Umemura ${ }^{8}$ proposed a supplementary "long-wave mode" mechanism to explain the breakup of a liquid jet issued from a nozzle. This mechanism incorporates interactions between upstream propagating capillary waves and the nozzle exit. Recently, filament pinch-off and breakup processes have been studied in relation to other applications, such as droplet formation in immiscible liquid-liquid systems, ${ }^{31}$ pinch-off locations in viscoelastic alginate solutions, ${ }^{32}$ capillary breakup of magnetic fluids, ${ }^{33}$ and electric field-induced pinch-off of a compound droplet. $^{3}$

Compared with droplet evolution from the ligament, studies on how the ligament is generated from the bulk liquid, which is different for various atomization conditions, are very limited. Interfacial instability and other analogous theories are usually used to determine whether liquid surface deformation can grow over time or atomization can occur. Thus, several practical atomization models have been proposed to predict atomization characteristics. For example, the WAVE model, which was constructed using the classical Kelvin-Helmholtz $(\mathrm{K}-\mathrm{H})$ instability, is now one of the most widely used breakup models for the simulation of spray in internal combustion engines. ${ }^{35}$ Rayleigh-Taylor (R-T) instability, which occurs on the interface when acceleration acts from a heavy fluid to a light one, has also been applied to build a high-pressure injection breakup model. ${ }^{36}$ Based on the theory of elasticity, O'Rourke and Amsden $^{37}$ analogized surface deformation to a mass-springdamper system and proposed the Taylor analogy breakup model to calculate atomized droplet sizes. Although these models have contributed greatly to industrial spray simulation, they still require a laborious, unphysical tuning process to match simulation results to experimental data because these models do not address the detailed dynamics of ligament formation.

In the present paper, we focus on liquid atomization caused by vertical mechanical vibration. Its basic mechanism is typically recognized as Faraday instability, which occurs on the surface of a bulk liquid that is subject to vertical vibration. ${ }^{38}$ In addition, Faraday instability has been reported by Umemura and Shinjo ${ }^{39}$ to be accounted for the local breakup caused by the vortices in the turbulent atomization. These turbulent vortices play the same role as the periodic external vibration. Many characteristics associated with Faraday instability, such as unstable liquid layer and spherical droplet boundaries, ${ }^{40-42}$ the pattern selection on the liquid surface, ${ }^{43-45}$ and the threshold acceleration for droplet ejection, ${ }^{15,19,46}$ have been studied via various experiments, simulations, and theories. However, as with other atomization, the highly nonlinear vibration-induced ligament formation mechanism has not yet been discussed thoroughly. As a result, the intrinsic physics of Lang's equation, ${ }^{24}$ which is a commonly used empirical equation that predicts the sizes of droplets formed via vibration-induced atomization, remain ambiguous. Furthermore, as pointed out by $\mathrm{Li}$ and Umemura $^{47}$ in a two-dimensional (2D) simulation of single-mode Faraday instability, ligament evolution can be mimicked as a lowspeed liquid jet issued from a nozzle under acceleration. This may bridge the liquid jet atomization theory to the vibration-induced atomization process. To the best of our knowledge, existing efforts to illuminate the analogy between these two atomization scenarios are limited. Thus, we perform the present three-dimensional (3D) simulation to study the detailed nonlinear dynamics associated with ligament formation and breakup caused by Faraday instability.

In the following, Sec. II describes the numerical schemes adopted in the present study and validation of numerical methodology. Then, ligament development, mechanisms of ligament formation and droplet breakup, and analogy between ligament development and low-speed jet breakup are thoroughly discussed in Sec. III. Finally, the conclusions of this research are summarized in Sec. IV.

\section{NUMERICAL METHODOLOGY AND VALIDATION}

\section{A. Numerical methods}

In the present study, the viscous effect of fluids is excluded to emphasize interactions between inertial and capillary forces. The conservation equations for incompressible, variable-density flow with surface tension for two-phase immiscible fluids under external vibration are

$$
\begin{gathered}
\partial_{t} \rho+\nabla \cdot(\rho \boldsymbol{u})=0, \\
\rho\left(\partial_{t} \boldsymbol{u}+\boldsymbol{u} \cdot \nabla \boldsymbol{u}\right)=-\nabla p+\rho \Delta_{0} \Omega^{2} \sin (\Omega t) \boldsymbol{j}+\sigma \kappa \delta_{s} \boldsymbol{n}, \\
\nabla \cdot \boldsymbol{u}=0,
\end{gathered}
$$

where $\boldsymbol{u}=(u, v, w)$ is the velocity vector (whose vertical component $v$ is relative to the vibrating bottom substrate), $p$ is the pressure, and $\rho(\boldsymbol{x}, t)$ is the density. The second term on the right-hand side of Eq. (2) represents the external vibration in the vertical direction $\boldsymbol{j}$, whose amplitude and angular frequency are $\Delta_{0}$ and $\Omega$, respectively. The surface tension is incorporated using the continuum-surfaceforce (CSF) method, ${ }^{48,49}$ coupled with a height-function curvature calculation. ${ }^{50,51}$ Correspondingly, the delta function $\delta_{s}$ indicates that the surface tension coefficient $\sigma$ is concentrated at the interface. The curvature of the interface is denoted by $\kappa$, while $\boldsymbol{n}$ is the unit outward vector normal to the interface. 
A volume-of-fluid function (VOF) $c(x, t)$ is introduced to trace the multiphase interface and calculated using the advection equation,

$$
\partial_{t} c+\nabla \cdot(c \boldsymbol{u})=0
$$

in which $c$ is the volume fraction, with $c=0$ and $c=1$ denoting gaseous and liquid phases, respectively. Naturally, $0<c<1$ indicates the gas-liquid interface. The density can thus be constructed as $\rho(c)=c \rho_{l}+(1-c) \rho_{g}$, with the subscripts $l$ and $g$ standing for liquid and gas properties, respectively. A staggered temporal discretization for the density/volume fraction and pressure leads to a scheme of second-order accuracy in time. ${ }^{52}$ A classical time-splitting projection method ${ }^{53}$ is used, which requires the solution of a Poisson equation. To improve the numerical efficiency and robustness, the discretized momentum equation is reorganized to the Helmholtztype equation, which can be solved via an improved multilevel Poisson solver. ${ }^{52}$ Spatial discretization is achieved by a graded Octree partitioning in three dimensions. All the variables are collocated at the center of each discretized cubic volume and are interpreted as volume-averaged values for each cell.

To solve the advection equation for the volume fraction, a piecewise-linear geometrical VOF scheme generalized for the Quad/Octree spatial discretization is employed. Furthermore, combination of balanced-force surface tension discretization and a height-function curvature estimation is employed to accurately capture the surface tension-driven flow. In the present study, we used the aforementioned schemes as implemented in the open source code Gerris. ${ }^{52,54}$

\section{B. Numerical specifications}

The computational domain and numerical specifications are illustrated in Fig. 1. For a single-mode calculation using wavelength $\lambda$, the domain is $5 \lambda$ in height with a rectangular cross section of $\lambda \times \lambda$. The sidewalls are specified with periodic boundaries, while the bottom and top boundaries had slip wall and free outflow boundary conditions, respectively. The liquid layer resting horizontally on the substrate is $1.5 \lambda$ in depth at rest to allow sufficiently large surface deformation since the main dynamics associated with ligament formation are limited to a depth of the reciprocal of the wavenumber $(1 / k)$ from the interface. ${ }^{47}$ To initiate Faraday instability, an initial displacement disturbance $\varepsilon_{0} \cos (k x) \cos (k z)$ is imposed on the interface as schematized in Fig. 1. The origin of the coordinate system is on the symmetric axis and $1.5 \lambda$ above the bottom surface.

To obtain a generalized understanding, the coordinates $(x$, $y, z)$ are normalized by the wavelength $\lambda=2 \pi / k$ and the time $t$ by the forcing frequency $f=\Omega / 2 \pi$ such that $\hat{x}, \hat{y}, \hat{z}=x, y, z / \lambda$ and $\hat{t}=t f$. Since the velocity field induced by the inertial force $\rho \Delta_{0} \Omega^{2} \sin (\Omega t)$ should depend on the forcing displacement amplitude $\Delta_{0}$ as well as the characteristic time scale $\Omega$, the dimensionless velocity $\hat{\boldsymbol{u}}=\boldsymbol{u} /\left(\Delta_{0} \Omega\right)$ and pressure $\hat{p}=p /\left(\rho_{l} \Delta_{0}^{2} \Omega^{2}\right)$ are adopted.

In the present study, the entire computational domain is composed of five boxes with length $\lambda$ that are arranged in the $y$ direction. To resolve the multiple scales involved in ligament formation and subsequent breakup accurately, the computation domain is divided into three physical zones: the gaseous medium, the liquid medium,

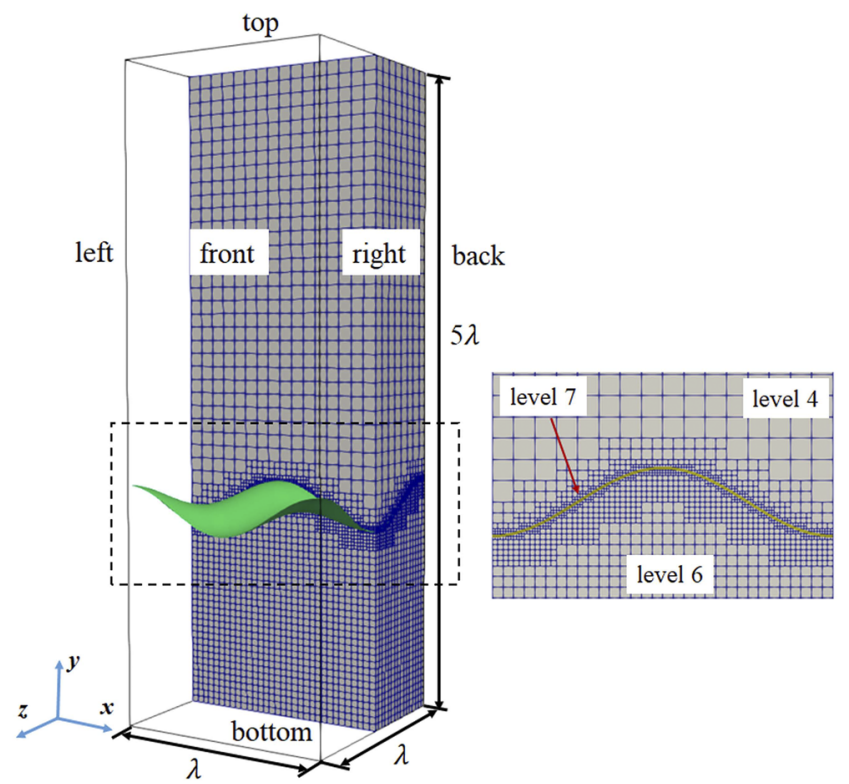

FIG. 1. Computational domain and numerical setup for the three-dimensional simulation (not to scale).

and the interface. Each zone has its own mesh refinement level denoted by $N$, which corresponds to a minimum mesh size of $\lambda / 2^{N}$. Accordingly, the set $\left(N_{g}, N_{l}, N_{i}\right)$ is used to specify the refinement levels in three zones. A typical three-dimensional simulation with mesh refinement levels $(4,6,7)$ results in 726115 grid cells throughout the domain. This is equivalent to $\sim 10^{8}$ grid cells for a uniform mesh of level 7. For the simulation with grid adaption, it takes about $120 \mathrm{~h}$ of real time to run a simulation to $\hat{t}=4.0$ on an Intel Xeon(R) Gold-5115 processor with 32 cores.

\section{Numerical validation and grid-convergence analysis}

To validate the present numerical setup, two benchmark simulations have been conducted and compared with both experiment of Jiang et al. ${ }^{55}$ and previous simulation results of Wright et al., ${ }^{56}$ respectively. In the experiment by Jiang et al., extra gravitational force and viscous damping are considered, and thus, the momentum equation is extended to

$$
\rho\left(\partial_{t} \boldsymbol{u}+\boldsymbol{u} \cdot \nabla \boldsymbol{u}\right)=-\nabla p+\left[\rho \Delta_{0} \Omega^{2} \sin (\Omega t)-g\right] \boldsymbol{j}+\sigma \kappa \delta_{s} \boldsymbol{n}-2 \beta \boldsymbol{u} .
$$

For the subharmonic mode, the corresponding physical parameters are $\rho_{l}=1000 \mathrm{~kg} \mathrm{~m}^{-3}, \rho_{g}=1 \mathrm{~kg} \mathrm{~m}^{-3}, \sigma=0.072 \mathrm{~N} \mathrm{~m}^{-1}, \Omega=20.55 \mathrm{rad}$ $\mathrm{s}^{-1}, \lambda=600 \mathrm{~mm}, \mathrm{~g}=9.81 \mathrm{~m} \mathrm{~s}^{-2}$, and $\Delta_{0}=2.65 \mathrm{~mm}$. The damping rate $\beta$ is set to $0.05 \mathrm{~s}^{-1}$ by following the experiment measurement. It should be noted that the experiment was conducted in a narrow rectangular tank of $600 \times 60 \times 483 \mathrm{~mm}^{3}$ with an operation water depth of $\sim 300 \mathrm{~mm}$. Because of this large aspect ratio (10:1), the wave field remained two-dimensional during the experiment. Consequently, $2 \mathrm{D}$ simulation is performed with grid refinement level set $(4,6,7)$ in the present work. As shown in Fig. 2, during the initial stage, 


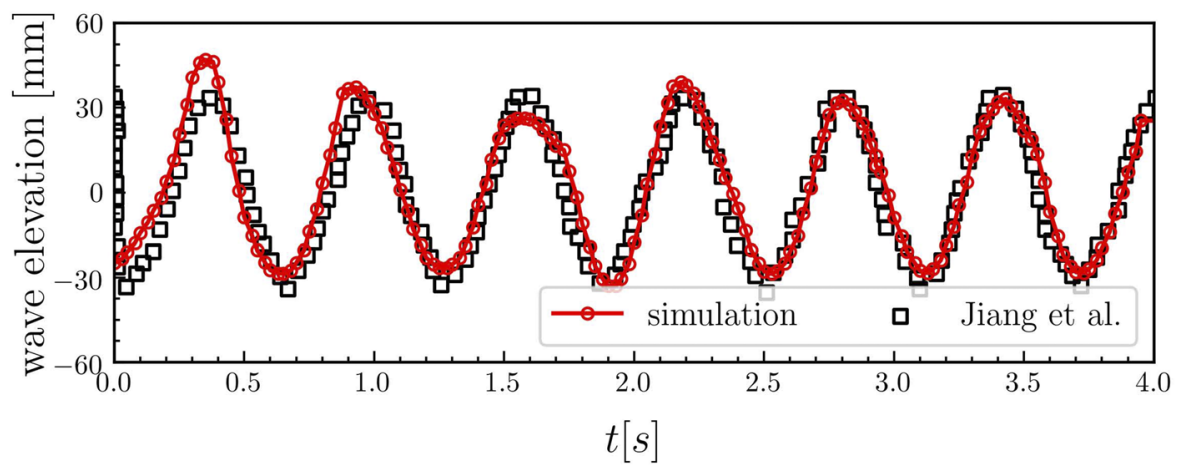

FIG. 2. Temporal evolution of the surface displacement at the interface of the present simulation compared against the experiment by Jiang et al. ${ }^{5}$

the simulation shows slight overshoot at the crests, while it agrees well with the experiment data at the troughs. As the initial transient dies down after $t=2 \mathrm{~s}$, very good agreement between the present simulation and experiment result is achieved.

For a straightforward comparison with a well-defined initial condition, we further validate our simulation with the $2 \mathrm{D}$ vortexsheet calculation by Wright et al. ${ }^{56}$ The physical parameters are $\rho_{l}=1000 \mathrm{~kg} \mathrm{~m}^{-3}, \rho_{g}=1 \mathrm{~kg} \mathrm{~m}^{-3}, \sigma=0.072 \mathrm{~N} \mathrm{~m}^{-1}, \Omega=0.26728 \mathrm{rad}$ $\mathrm{s}^{-1}, \lambda=1.0 \mathrm{~m}$, and $\Delta_{0}=0.140 \mathrm{~m}$. This simulation is implemented without gravitational force and viscous damping, which is exactly the same form of Eq. (2). Initially, the interface between two fluids is perturbed as $\varepsilon_{0} \cos (k \hat{x})$ with $\varepsilon_{0}=0.01$.

Figure 3 presents the temporal evolution of the surface displacement at $\hat{x}=0$, where the ligament eventually formed. The surface deformation implies a sub-harmonic mode, and the overall trend agrees well with the result by Wright et al. ${ }^{56}$ Furthermore, a grid convergence study was conducted with three grid refinement level combinations $(4,5,6),(4,6,7)$, and $(4,7,8)$. It can be observed from Fig. 3 that the discrepancies between the present simulation and the calculation by Wright et al. diminished as the grid resolution improved. Quantitatively, the relative error between the maximum displacements predicted by interface refinement levels 7 and 8 is

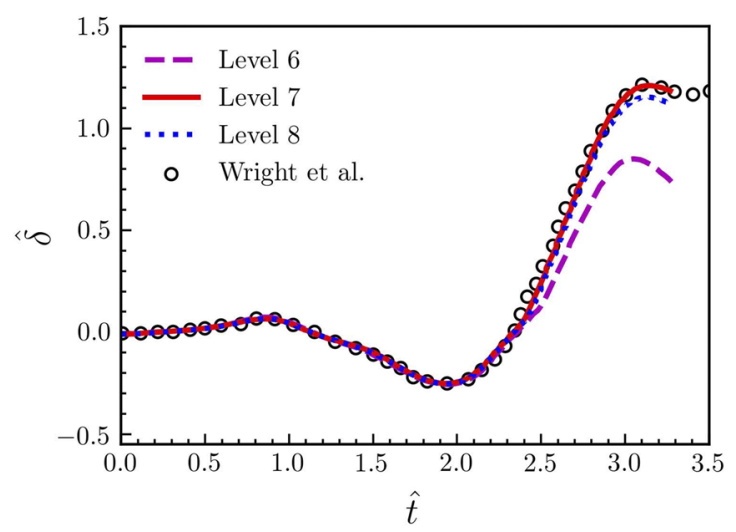

FIG. 3. Temporal evolution of the surface displacement at $\hat{x}=0$ obtained via the present simulation with various refinement levels and compared to the result from Wright et al. ${ }^{5}$ within $4 \%$. Since the similar characteristic dimensionless parameters are to be used in the $3 \mathrm{D}$ scenario, it is natural to employ the grid refinement level $(4,6,7)$ in subsequent simulations as a balance between the numerical accuracy and computational cost.

\section{RESULTS AND DISCUSSION}

In the present study, we intend to investigate the nonlinear dynamics associated with ligament formation on the liquid surface and breakup caused by external vibration. Although the surface response discussed in the theoretical work by Benjamin and Ursell $^{41}$ varies with the vibration conditions, we are quite interested in the sub-harmonic regime of the liquid surface oscillation, which is most commonly observed in realistic experiments. The interfacial instability caused by external vibration of inviscid fluids is characterized by two dimensionless parameters: $X=k \Delta_{0}$ and $Y=\sigma k^{3} / \rho_{l} \Omega^{2}$. In the present study, we mainly consider the case of $(X=0.88$, $Y=0.25)$, which can be recognized as the real condition, wherein $\rho_{l}=1000 \mathrm{~kg} \mathrm{~m}^{-3}, \rho_{g}=1 \mathrm{~kg} \mathrm{~m}^{-3}, \sigma=0.072 \mathrm{~N} \mathrm{~m}^{-1}, \Omega=6.28$ $\times 10^{3} \mathrm{rad} \mathrm{s}^{-1}, \lambda=1.219 \mathrm{~mm}$, and $\Delta_{0}=0.171 \mathrm{~mm}$, as the prototype for illustration of basic ligament development and droplet generation mechanisms. This combination of $X$ and $Y$ implies a large surface deformation and ligament formation with a sub-harmonic surface oscillation response. Other cases with different combinations of $X$ and $Y$ are also simulated, and their results will be demonstrated in corresponding discussions.

\section{A. General surface development dynamics}

The temporal evolution of the liquid surface and the liquidphase velocity field are depicted in Fig. 4, which demonstrates the slices on the center plane in the $z$ direction. Initially, a cosinusoidal deformation $\left[\varepsilon_{0} \cos (k x) \cos (k z)\right]$ with an amplitude of $\varepsilon_{0}=0.1 \lambda$ is imposed on the surface as the displacement disturbance, and the entire system is placed in a stagnant state. It is clearly shown that the surface deformation oscillates at half of the vibration frequency. Since the initial deformation displacement is relatively large, surface deformation development quickly becomes nonlinear. According to the study by $\mathrm{Li}$ and Umemura, ${ }^{47}$ nonlinear dynamics predominate when the surface deformation amplitude exceeds $0.1 \lambda$, which is the initial amplitude of the disturbance in the present study. As a result, the surface deformation loses its initial cosinusoidal shape quickly. 


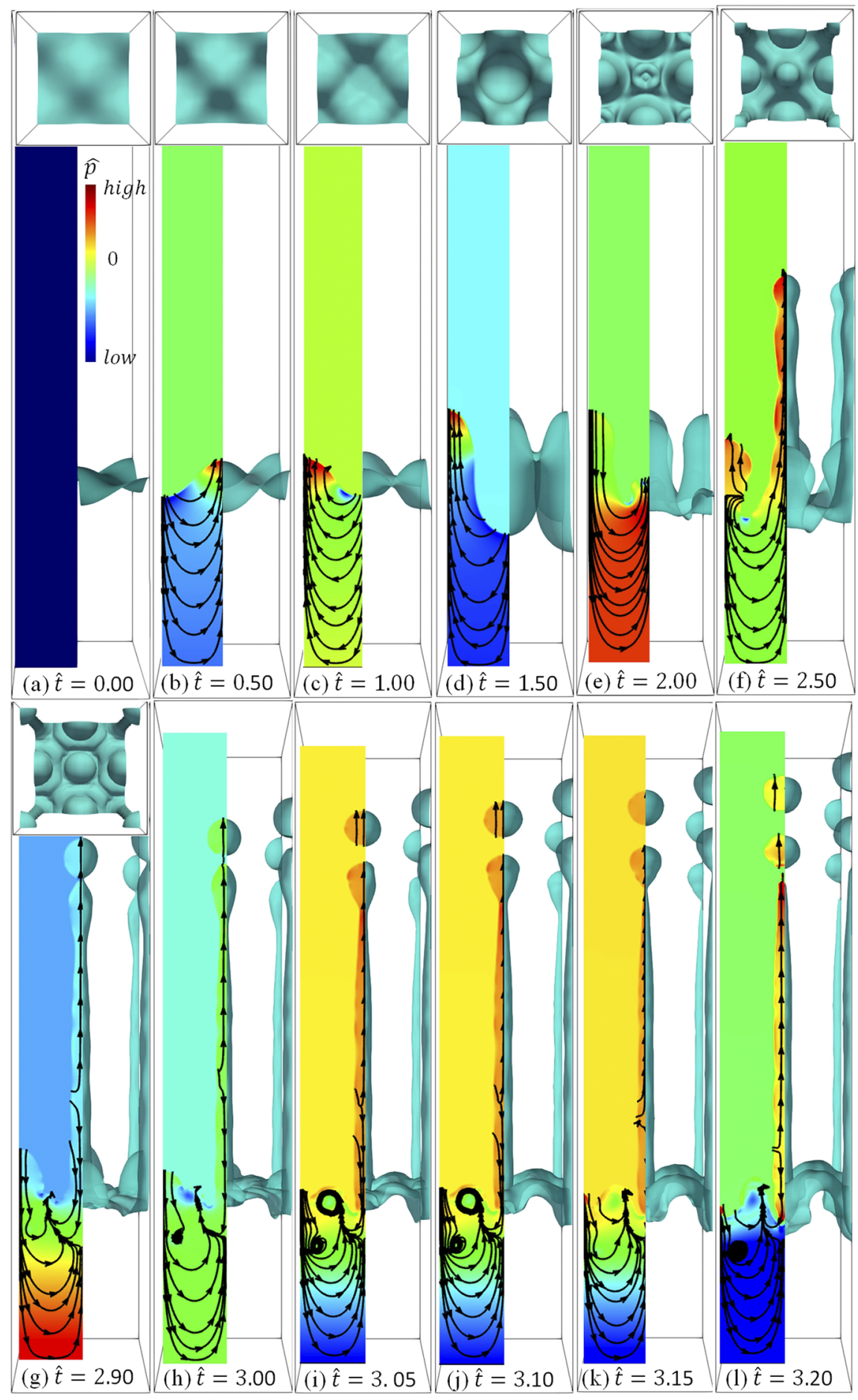

FIG. 4. Temporal evolution of liquid surface deformation (right half of each frame) along with pressure contours and streamlines (the vertical component of velocity vector is the one relative to the bottom substrate) viewed on the center plane in the $z$ direction (left half of each frame). The corresponding time instances of (a)-(I) are denoted in each sub-frame. The top views are shown at the top of each frame until $\hat{t}=2.90$ as those of frames $(h)-(l)$ are similar to frame $(\mathrm{g})$.

Following the treatment adopted by $\mathrm{Li}$ and Umemura, ${ }^{47}$ we divide one external vibrating period into two phases: the destabilizing phase when $X \sin (2 \pi \hat{t})-Y>0$ and the stabilizing phase when $X \sin (2 \pi \hat{t})-Y \leq 0$. The phases are separated based on whether the surface displacement amplitude growth rate is positive over time in the Mathieu equation. ${ }^{41}$ For the parameter combination $(X=0.88$, $Y=0.25)$ adopted herein, the destabilizing phase occurs when $(0.046+n)<\hat{t}<(0.454+n)$, where $n=0,1,2, \ldots$ This is highlighted 


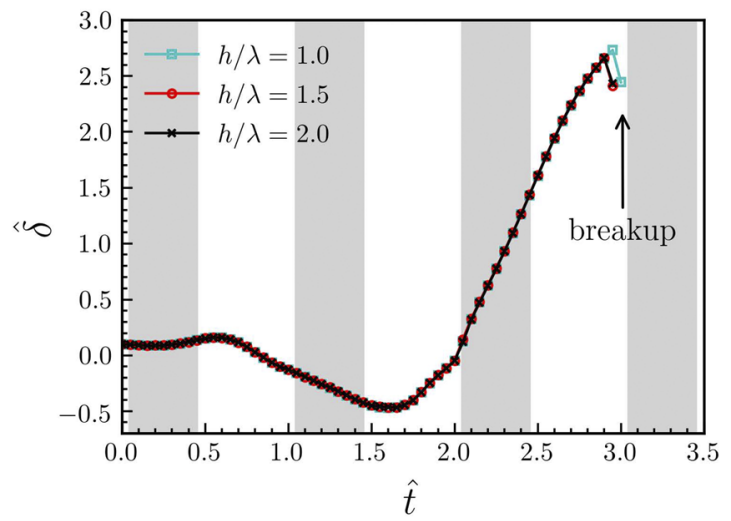

FIG. 5. Temporal evolution of the surface displacement at the tip of the crest centerline $(x=0, z=0)$ along the $y$ axis. The gray regions represent destabilizing phases. The effect of the liquid depth can be neglected if $h / \lambda \geq 1.5$.

via the gray regions in Fig. 5, which shows the temporal evolution of the surface displacement at the centerline along the $y$ axis.

From Fig. 5, we can see that the surface displacement amplitude grows slightly during the first destabilizing phase and faster during the second destabilizing phase. Due to the large initial surface disturbance and relatively fierce external vibration, in this case, the crest displacement grows rapidly to form a ligament during the third destabilizing phase. Note that the surface displacement continues to grow after $\hat{t}=2.454$ because of inertia. When the ligament is formed, its tip is rounded due to surface tension contraction, and capillary waves can be clearly seen on the surface of the ligament as shown in Fig. 4(f). Upon elongation of the surface ligament, the first neck on the ligament pinches off, and the first breakup from the ligament tip occurs at approximately $\hat{t}=2.95$. Next, as shown in Figs. 4(h)-4(1), a new rounded tip is formed, and the second neck continues thinning to produce subsequent breakups at $\hat{t}=3.2$.

Except for the front part of the ligament growth, the root region of the ligament connected to the bottom bulk liquid layer exhibits interesting phenomena. Figures $4(\mathrm{~g})-4(\mathrm{l})$ show that the ligament root evolves from an upwardly converging column to a straight cylinder as time elapses. Consequently, both the curvature and its resultant capillary pressure increase. These changes lead to detachment of the ligament from the bottom bulk liquid layer to form a separated ligament. This is different from the results obtained via $2 \mathrm{D}$ simulation, ${ }^{47}$ in which the converging shape is retained throughout the ligament formation process. The detailed dynamics and mechanisms of ligament formation and breakup will be discussed in Secs. III B-III D.

\section{B. Ligament formation mechanisms}

Ligament formation is the transitional stage of droplet generation from a liquid layer subject to external vertical vibration. It is associated with highly nonlinear dynamics because linear theories cannot predict the slender liquid ligament shape. In this section, the dynamics of how a large surface crest develops to extrude toward the gas phase and form a slender ligament will be elaborated.
Subsequent successive breakups are discussed based on detailed pressure and velocity field information.

Figure 6 shows the temporal evolution of a surface crest to form a liquid ligament during the third destabilizing phase from $\hat{t}=2.0$ to $\hat{t}=2.5$ using fine time steps. Each frame depicts the pressure contour and streamline (left half of each frame in Fig. 6) alongside surface deformation (right half of each frame in Fig. 6). To determine how nonlinear effects deform the bumped crest into a slender ligament, we need to scrutinize the dynamic structure of the severely deformed surface. Detailed vertical velocity and pressure distributions along the central line of the crest $(x=0, z=0)$ during this period are shown in Figs. 7 and 8, respectively. We can use these to divide the entire liquid field into three sub-regions with different flow characteristics.

Figure 7 shows that the vertical velocity relative to the bottom substrate is almost zero away from the deformed surface, which indicates that movement of the liquid in this region is synchronized with that of the bottom substrate. This is because the effect of a large deformation reaches a limited depth from the surface on the order of $1 / k^{47}$ In fact, the pressure in the region away from the surface distributes almost identical at the same level, and, as shown in Fig. 8, vertical pressure gradients $(\partial p / \partial y)$ at different times coincide with the external vibration acceleration (the thick gray lines in Fig. 8). Thus, quasi-stagnant flow away from the liquid surface plays no significant role in ligament formation.

In the region near the surface, the most important dynamic is the appearance of a local maximum pressure point. This is a typical result caused by the nonlinear effect. As shown in Fig. 6, the horizontal liquid flow from the adjacent trough portions collides at the root of the crest and enhances the pressure there. This pressure enhancement at the root of the crest prevents the liquid flowing into the crest region to increase the crest volume. This is different from the flow structure in the linear regime, in which the pressure at the crest root decreases as the crest height increases and hence additional liquid is sucked from adjacent trough portions to further increase the crest height. Thus, this nonlinear flow structure retards crest height development. Another effect of pressure enhancement is formation of local maximum pressure points shown as solid black circles in the inset of Fig. 8. The liquid region above this point is dynamically freed from the bulk liquid region below it. Once the local maximum pressure point is formed, the crest portion above it elongates freely to form a slender ligament.

The liquid region above the local maximum pressure point exhibits free development behavior, as shown in Fig. 8. It is clear that the vertical pressure gradient $(\partial p / \partial y)$ approaches zero in the ligament, and the pressure magnitude is rather small except in the tip region, where its bulbous shape induces capillary pressure. Thus, the liquid flow above the local maximum pressure point is not affected by bottom vibration. The liquid elements that enter the ligament region are "freed" by the maximum pressure point and retain their velocities in the laboratory coordinate system. This free ligament development continues as its height (length) increases until breakup occurs at the tip due to the interfacial instability that will be discussed in Sec. III C.

\section{Droplet formation mechanism}

After a slender ligament is formed, a capillary wave develops on the ligament surface, and the ligament eventually breaks up to 


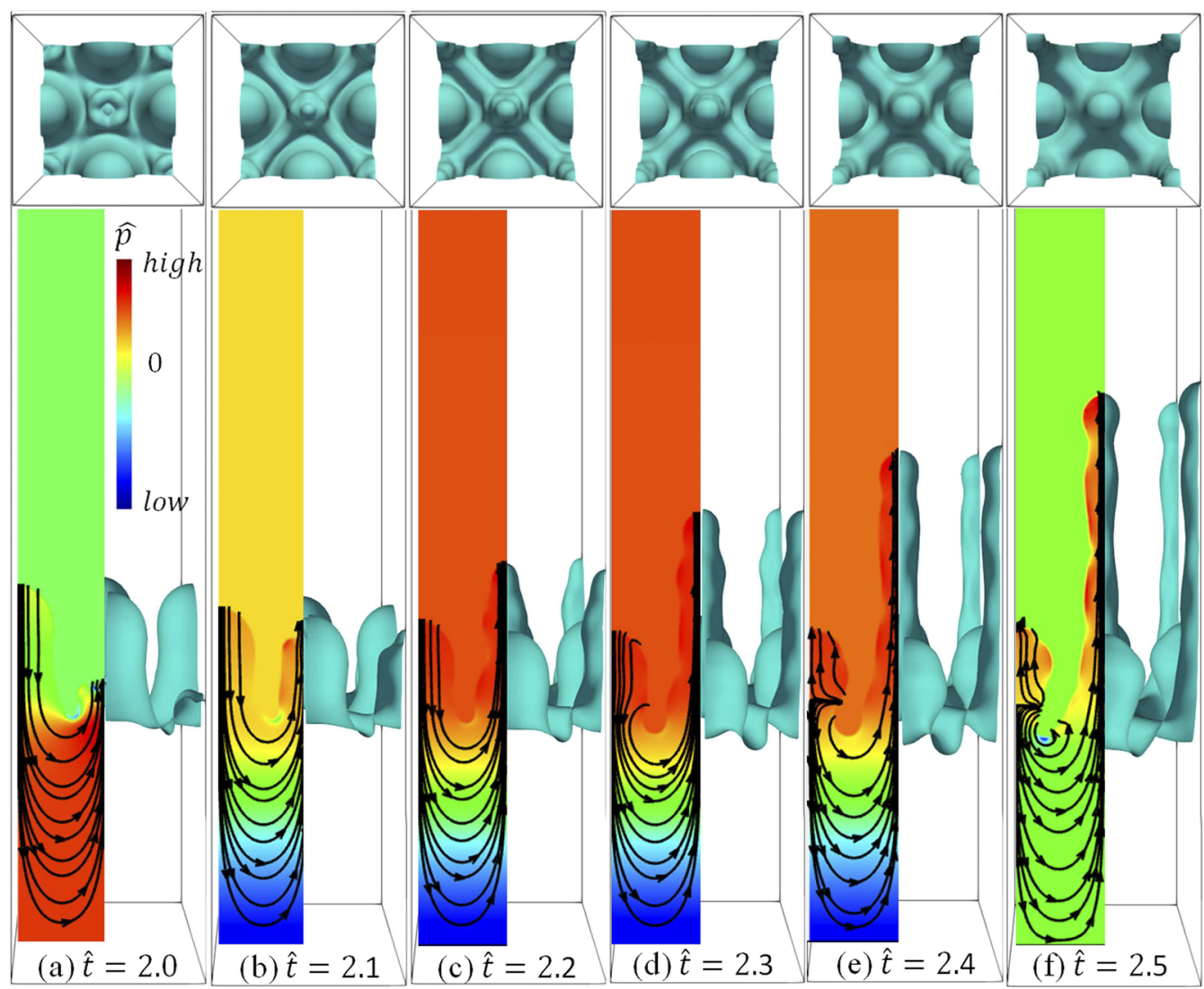

FIG. 6. Temporal evolution of the surface crest to form a liquid ligament during the third destabilizing phase from $\hat{t}=2.0$ to $\hat{t}=2.5$ as (a)-(f) with a time interval of 0.1 . The top views are shown at the top of each frame.

generate multiple droplets due to capillary instability. In this section, we will elaborate the characteristics of capillary waves and the mechanism by which instability causes ligament breakup and droplet formation.
Figure 9 shows the temporal evolution of the capillary wave on liquid ligament from $\hat{t}=2.5$ to $\hat{t}=3.0$ using fine time steps. The first pinch-off occurs during this period, and the resulting droplet is generated from the ligament tip. The associated pressure and

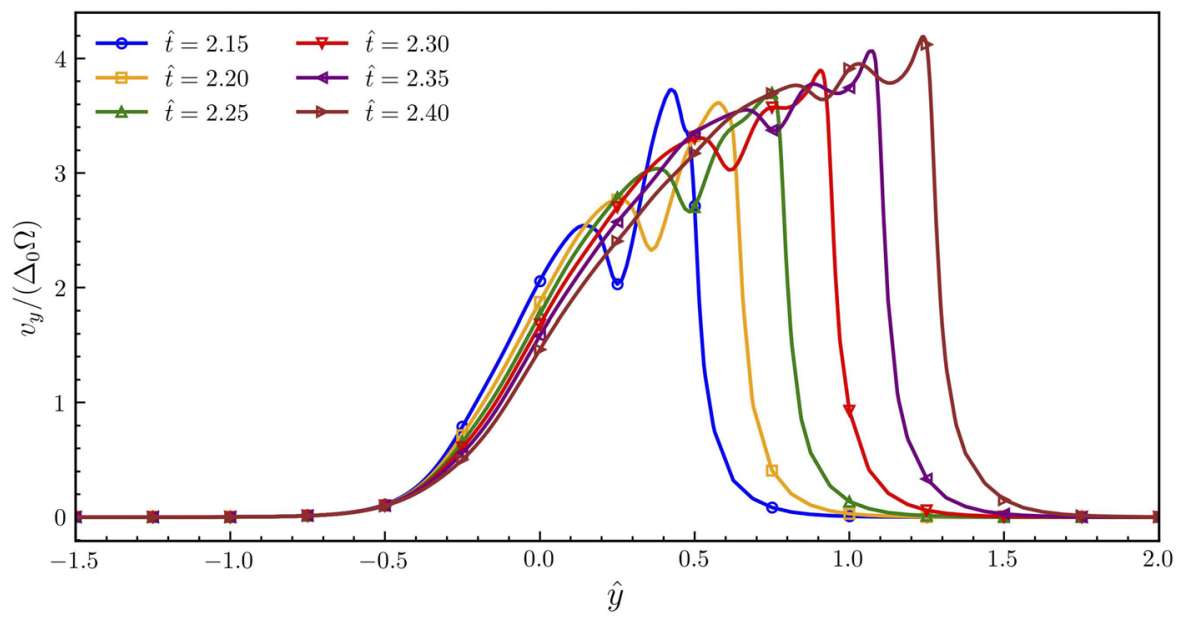

FIG. 7. Distributions of vertical velocities relative to the bottom substrate along the crest centerline $(x=0, z=0)$ during ligament evolution. 


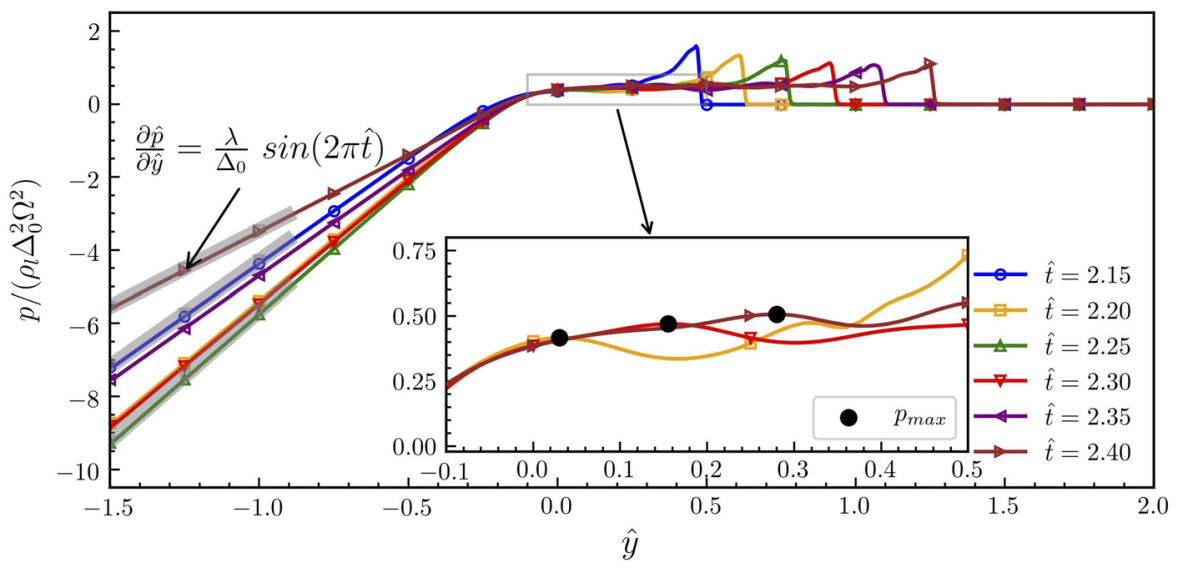

FIG. 8. Pressure distributions along the crest centerline $(x=0, z=0)$ during ligament evolution. The thick gray lines in the bottom region represent the dimensionless external acceleration. The solid black circles in the inset chart represent local maximum pressure points near the ligament root.

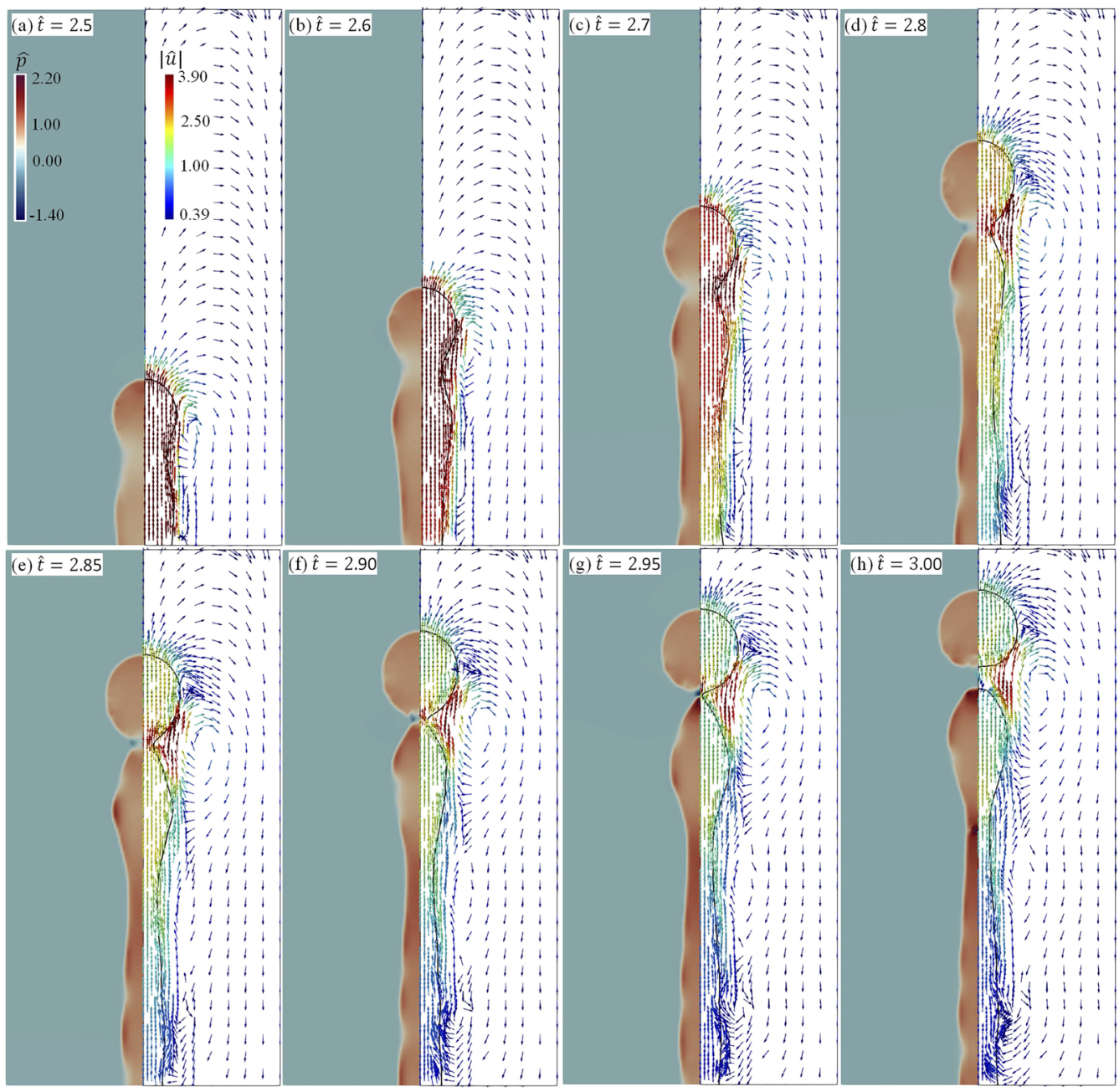

FIG. 9. Formation of the first droplet during $2.5 \leq \hat{t} \leq 3.0$. (a)-(d) indicate the time range from 2.50 to 2.80 with a time interval of 0.1 . (e)-(h) indicate the time range from 2.85 to 3.00 with a time interval of 0.05 . The left half shows the pressure contour while the right half presents the velocity vector colored by its magnitude. The bottom boundary of each frame is $\lambda$ away from the origin. 
velocity distributions are depicted as contours and vectors, respectively. On one hand, the ligament is elongated because of continuous liquid flow from the bottom bulk to the ligament region. On the other hand, the capillary wave generated due to contraction of the ligament tip propagates upstream (opposite to the direction of ligament growth) along the ligament. The capillary wave is a dispersive wave whose phase speed is inversely proportional to its wavelength. Thus, a capillary wave with a small wavelength propagates quickly toward the ligament root. In the present prototype case, the capillary wave reaches a quasi-steady-state wavelength of $3.64 a$ (where $a \approx 0.1 \lambda$ is the mean radius of the ligament) and increases its amplitude until it breaks up at the first neck portion.

This quasi-steady behavior of capillary wave on a ligament is similar to a free liquid thread with radius $a$ under capillary force, which has been derived by Umemura ${ }^{8,57}$ using a simplified onedimensional (1D) model as shown in Fig. 10. The theoretical formulations and solutions following Umemura's derivations are detailed in Appendixes $\mathrm{A}$ and $\mathrm{B}$ for reference. According to this theory (see Appendix A), the ligament tip contracts at a constant velocity $U=\sqrt{\sigma / \rho a}$, and this contraction would generate a steady capillary wave traveling upstream of ligament with the wavelength of $\Lambda=\frac{2 \pi}{\sqrt{3}} a \approx 3.63 a$ (see Appendix $B$ ), which agrees well with the numerical result of $3.64 a$ for the prototype case $(X=0.88, Y=0.25)$.

Besides the prototype case, other simulations with different parameter combinations that can realize breakup are additionally conducted to study their effects on the characteristics of the ligament development. The resultant quasi-steady capillary wavelengths for these cases are shown in Table I. It shows that although the ligament radius and capillary wavelength vary with different parameter combinations, the ratio of capillary wavelength to ligament radius, $\Lambda / a$, changes relatively little. The deviation of $\Lambda / a$ from 3.63 derived for the microgravity case (Appendix B) ${ }^{57}$ is considered to be caused by a temporally periodic acceleration acting on the ligament

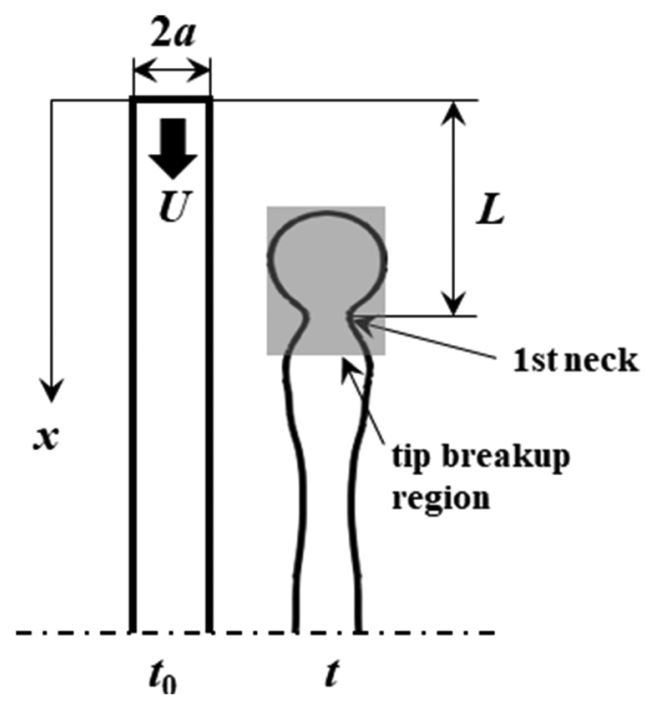

FIG. 10. Schematic of the one-dimensional model of tip contraction and capillary wave development in a liquid thread.
TABLE I. Ligament radius, quasi-steady capillary wavelength, and effective diameter of disintegrated droplets at the ligament tip for simulations of different parameter combinations.

\begin{tabular}{lccc}
\hline \hline Cases & $a$ & $\Lambda$ & $D$ \\
\hline$(\boldsymbol{X}=0.80, \boldsymbol{Y}=0.25)$ & $0.135 \lambda$ & $3.73 a$ & $0.328 \lambda$ \\
$(\boldsymbol{X}=0.86, \boldsymbol{Y}=0.25)$ & $0.091 \lambda$ & $3.56 a$ & $0.336 \lambda$ \\
$(\boldsymbol{X}=0.88, \boldsymbol{Y}=0.25)$ & $0.100 \lambda$ & $3.64 a$ & $0.320 \lambda$ \\
$(\boldsymbol{X}=0.92, \boldsymbol{Y}=0.25)$ & $0.106 \lambda$ & $3.57 a$ & $0.375 \lambda$ \\
$(\boldsymbol{X}=0.86, \boldsymbol{Y}=0.22)$ & $0.089 \lambda$ & $3.33 a$ & $0.322 \lambda$ \\
\hline \hline
\end{tabular}

development in Faraday instability. This temporally periodic acceleration would affect the tip contraction dynamics of ligament and further influence the capillary wave behaviors.

In the coordinate system attached to the vibrating substrate, the dynamical behavior of the ligament caused by Faraday instability is mimicked by that of a liquid jet issuing from an orifice under a temporally periodic acceleration. Umemura et al. ${ }^{58,59}$ have studied the disintegration process of a vertically downward water jet under normal gravity and proposed a theoretical derivation to describe the tip contraction and breakup dynamics. Extending their theoretical framework, ${ }^{58}$ we treat the tip contraction behavior of a jet under a temporally periodic acceleration, which is detailed in Appendix C. The "+" and "-" signs on the right-hand side of expression (C7) represent that the tip contracts upstream and extends downstream, respectively.

The tip contraction speeds obtained by Eq. (C7) and a 3D numerical simulation on a liquid jet for the same condition of $\gamma_{0}=0.5$ and $\tilde{\Omega}=1$ ( $\gamma_{0}$ and $\tilde{\Omega}$ are defined in Appendix C) are shown in Fig. 11. It can be seen that after a short transition time of $\tilde{t} \sim O(1)$, the temporal evolution of tip contraction speed calculated from Eq. (C7) agrees well with the 3D simulation result quantitatively, which validates the model derived in Appendix C. This also indicates that, since the development of the Faraday-type ligament's

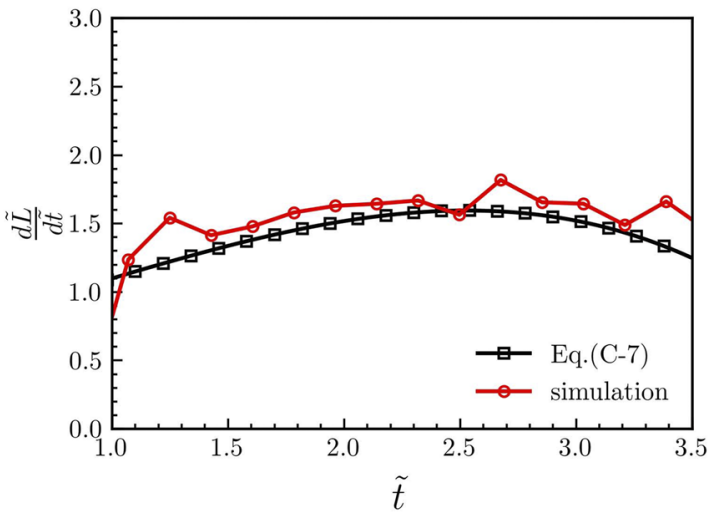

FIG. 11. Comparison between the tip contraction speeds $d \tilde{L} / d \tilde{t}$ of a liquid jet under temporally periodic accelerations obtained from Eq. (C7) and the $3 \mathrm{D}$ numerical simulation under the same condition of $\gamma_{0}=0.5$ and $\tilde{\Omega}=1$. 
tip shares the same behaviors as the liquid jet under temporally periodic acceleration, the tip contraction dynamics of the Faraday-type ligament are better predicted by the model in Appendix $\mathrm{C}$ than those by the microgravity model with a constant contraction speed.

The effects of $\gamma_{0}$ and $\tilde{\Omega}$ on the tip contraction speed solved from Eq. (C7) are shown in Fig. 12. For the limit case of $\tilde{\Omega}=0$ (no oscillation), the problem reduces to the jet development under a constant acceleration. For the limit case of $\gamma_{0}=0$ at all $\tilde{\Omega}$-values, $d \tilde{L} / d \tilde{t}$ reaches a steady value of 1.0 rapidly after a short transition time, which reduces to the problem of jet development in microgravity that has been discussed in Appendix A. For the constant acceleration cases, as shown in Fig. 12(a), $d \tilde{L} / d \tilde{t}$ reaches its peak value and becomes zero earlier with the increase in $\gamma_{0}$, which indicates that the tip bulb would not push the upstream liquid after a certain time if the constant acceleration directing downstream of jet is considered.

When the effect of $\tilde{\Omega}$ is included, as shown in Figs. 12(b) and 12 (c), $d \tilde{L} / d \tilde{t}$ and $\tilde{L}$ become oscillatory over time. The tip contraction will be strengthened when the temporally periodic acceleration directs upstream of jet. As a result, different from the constant acceleration case, the tip contraction speed $d \tilde{L} / d \tilde{t}$ could exceed 1.0. The oscillation amplitude of $d \tilde{L} / d \tilde{t}$ increases with the increase in $\gamma_{0}$. According to Eq. (B10), the $\gamma_{0}$-dependent tip contraction speed for the temporally periodic acceleration case would influence the capillary wavelength, which is considered to be accountable for the varied $\Lambda / a$ on the Faraday-type ligaments at different parameter combinations.

It is noticeable from Fig. 12 that the temporal evolutions of tip contraction displacement $\tilde{L}$ for different $\gamma_{0}$-values converge to the straight line obtained in $\gamma_{0}=0$ with the increase in $\tilde{\Omega}$ and decrease in $\gamma_{0}$. This convergence indicates that the average value of jet tip contraction speed under a temporally periodic acceleration with high frequency and low amplitude approaches the steady value of $U=\sqrt{\sigma / \rho a}$.

The quasi-steady capillary wave on the ligament continues to develop, and the first neck pinches off to form a droplet from the ligament tip. It is well known that the Rayleigh-Plateau (R-P) instability, which is typically used to explain the breakup of an infinite liquid column, occurs only if the wavelength on the column surface is larger than its circumference of $2 \pi a$. The quasi-steady capillary wavelengths due to tip contraction for all simulated cases, as shown in Table I, are smaller than $4.0 a$, which is much smaller than the critical value for R-P instability to occur. Thus, breakup at the ligament tip is caused by another mechanism known as "short-wave mode" breakup instability.

The key to the short-wave mode breakup mechanism is the presence of a ligament tip, from which bulbs and necks are alternatively distributed when a steady capillary wave is formed on the ligament. We focus on the region (gray region in Fig. 10) around the first neck upstream the tip bulb (first bulb) to analyze the breakup process. Figures 13 and 14 show the distributions of pressure and vertical velocity in the laboratory coordinate system along the crest centerline at various time instances. The pressure in the downstream edge of the second bulb (left sides of the thick gray regions in Figs. 13 and 14) is higher than in the first neck due to interactions between circumferential and axial capillary forces. This results in a fast liquid flow that jets into the tip bulb through the neck region.
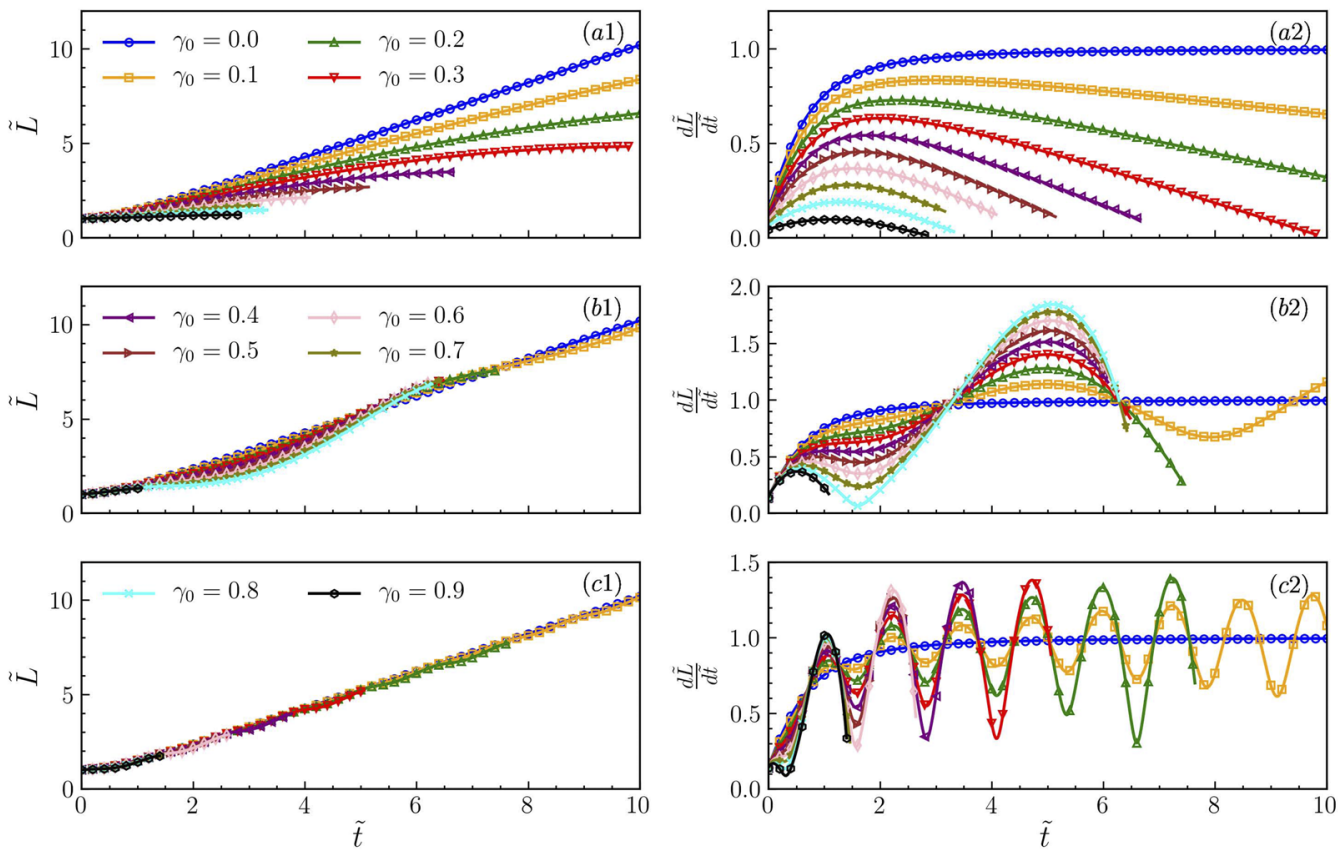

FIG. 12. Temporal evolutions of the tip contraction displacement $\tilde{L}$ and speed $d \tilde{L} / d \tilde{t}$ by solving Eq. (C7) with different values of $\gamma_{0}$ ranging from 0 to 0.9 for (a) $\tilde{\Omega}=0$, (b) $\tilde{\Omega}=1$, and (c) $\tilde{\Omega}=5$. 


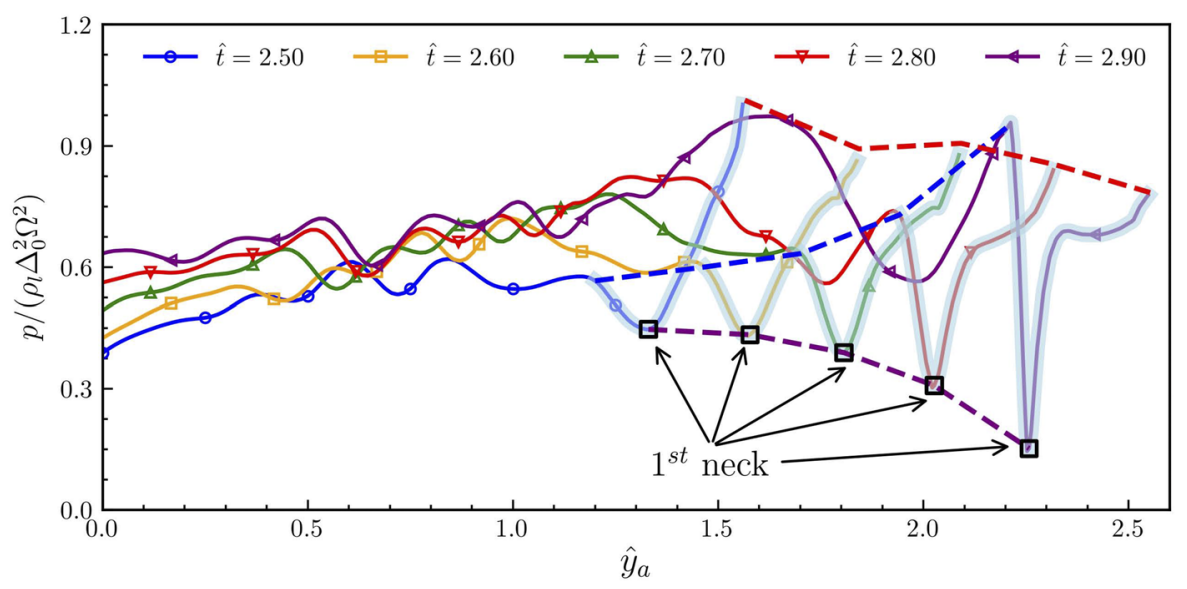

FIG. 13. Pressure distributions in the liquid phase along the crest centerline $(x=0, z=0)$ during droplet formation at vertical location $0.0 \leq \hat{y}_{a} \leq 2.5$. The thick gray lines denote the tip breakup region identified in Fig. 10. The red dashed line represents the pressure at the ligament tip. The pressure difference between the second bulb (blue dashed line) and the first neck (purple dashed line) increases over time, which enhances the jetted flow from the neck to the tip bulb.

The velocity reaches its maximum when the pressure is locally minimized in the neck region. This jetted liquid flow decelerates due to the relatively high pressure in the tip bulb region and accumulates to enlarge the bulb volume, which decreases the pressure in the tip bulb region (see the red dashed line in Fig. 13). The pressure difference between the second bulb (blue dashed line in Fig. 13) and first neck (purple dashed line in Fig. 13) increases over time. This continuously squeezes liquid from the neck region to the tip bulb, and thus, the first neck continues to pinch until breakup occurs at $\hat{t}=2.95$.

The size of the droplet disintegrated from the ligament tip is an important characteristic of the atomization caused by Faraday instability. The effective droplet diameter in the simulation of the prototype case is $D=3.2 a$. Since the mean ligament radius $a \approx 0.1 \lambda$, we can easily obtain

$$
D=3.2 a \approx 0.32 \lambda,
$$

which relates the size of a droplet formed by Faraday instability to the surface wavelength. The mean diameters of disintegrated droplets for other simulation cases are shown in Table I. It is shown that the ratio of droplet diameter to surface wavelength, $D / \lambda$, ranges narrowly between 0.32 and 0.375 for the present simulation cases, which are close to the empirical constant value of 0.34 reported in most previous experimental studies on the statistically averaging diameters of atomized droplets. ${ }^{24}$

According to the "short-wave mode" breakup theory, ${ }^{60}$ the size of the generated droplet depends on the volume $(\Delta V)$ entering the tip bulb during the period between the newly formed jet and breakup at the first neck. $\Delta V$ is a variable determined by the base flow of the ligament. The resultant diameter of the disintegrated droplet at the ligament tip caused by this "short-wave mode" breakup mechanism ranges from $2.8 a$ and $4.0 a{ }^{60}$ Once the dependence of the ligament radius $a$ on the surface wavelength $\lambda$ can be determined in possible future works, the empirical coefficient 0.34 in Lang's equation ${ }^{24}$ will be interpreted in a more physical fashion.

\section{Analogies with low-speed jet breakup}

From the above analyses, it is obvious that the morphologies of ligament development and breakup are quite similar to the behavior of a low-speed liquid jet issuing from a nozzle or orifice under a

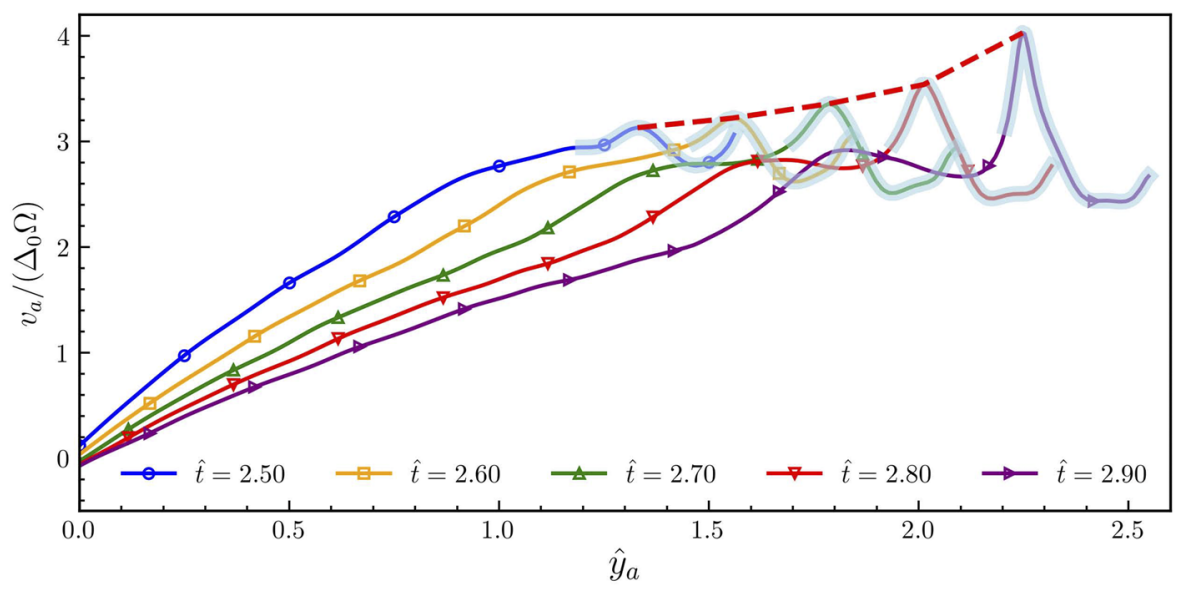

FIG. 14. Vertical velocity distributions (in the laboratory coordinate system) in the liquid phase along the crest centerline $(x=0, z=0)$ during droplet formation at vertical location $0.0 \leq \hat{y}_{a} \leq 2.5$. The thick gray lines denote the tip breakup region identified in Fig. 10. The red, dashed line represents the temporal tendency of the local maximum velocity in the first neck region. 
temporally periodic acceleration. In this section, an analogy between ligament development caused by Faraday instability and low-speed jet breakup under a temporally periodic acceleration will be demonstrated. This analogy may inspire efforts to bridge these two breakup regimes even though they have always been treated separately in previous studies.

As discussed in Sec. III B, the maximum pressure point formed in the crest root region is recognized as the indicator of freed ligament formation. Subsequent ligament development above the maximum pressure point is independent of external vibrations. As a result, the maximum pressure point can be treated as the source of liquid supply for ligament growth.

To further address the similarity and difference between the ligament development and breakup caused by Faraday instability and jet breakup, we conducted simulations on the evolution of a liquid jet issuing from an orifice with a constant speed under a temporally periodic acceleration. In the jet issue condition corresponding to the present prototype case of Faraday instability, the equivalent We number $W e=\rho_{l} V_{i n j}{ }^{2} a / \sigma=\rho_{l}\left(2 \Delta_{0} \Omega\right)^{2} a / \sigma \sim 8.0$, Bond number $B o=\rho_{l} A_{0} a^{2} / \sigma=\rho_{l} \Delta_{0} \Omega^{2} a^{2} / \sigma \sim 1.4$, and dimensionless angular frequency $\tilde{\Omega}=\Omega a / \sqrt{\sigma / \rho a} \sim 1.0$, where $A_{0}$ is the amplitude of temporally periodic acceleration and $V_{i n j}$ is the issuing speed of jet at orifice exit. $V_{i n j}=2 \Delta_{0} \Omega$ is determined by the vertical velocity at the maximum pressure point when it first emerges in the Faraday-type ligament regime.

The simulation results of this jet issue condition are exhibited in Fig. 15, as the counterpart of Faraday-type ligament development.

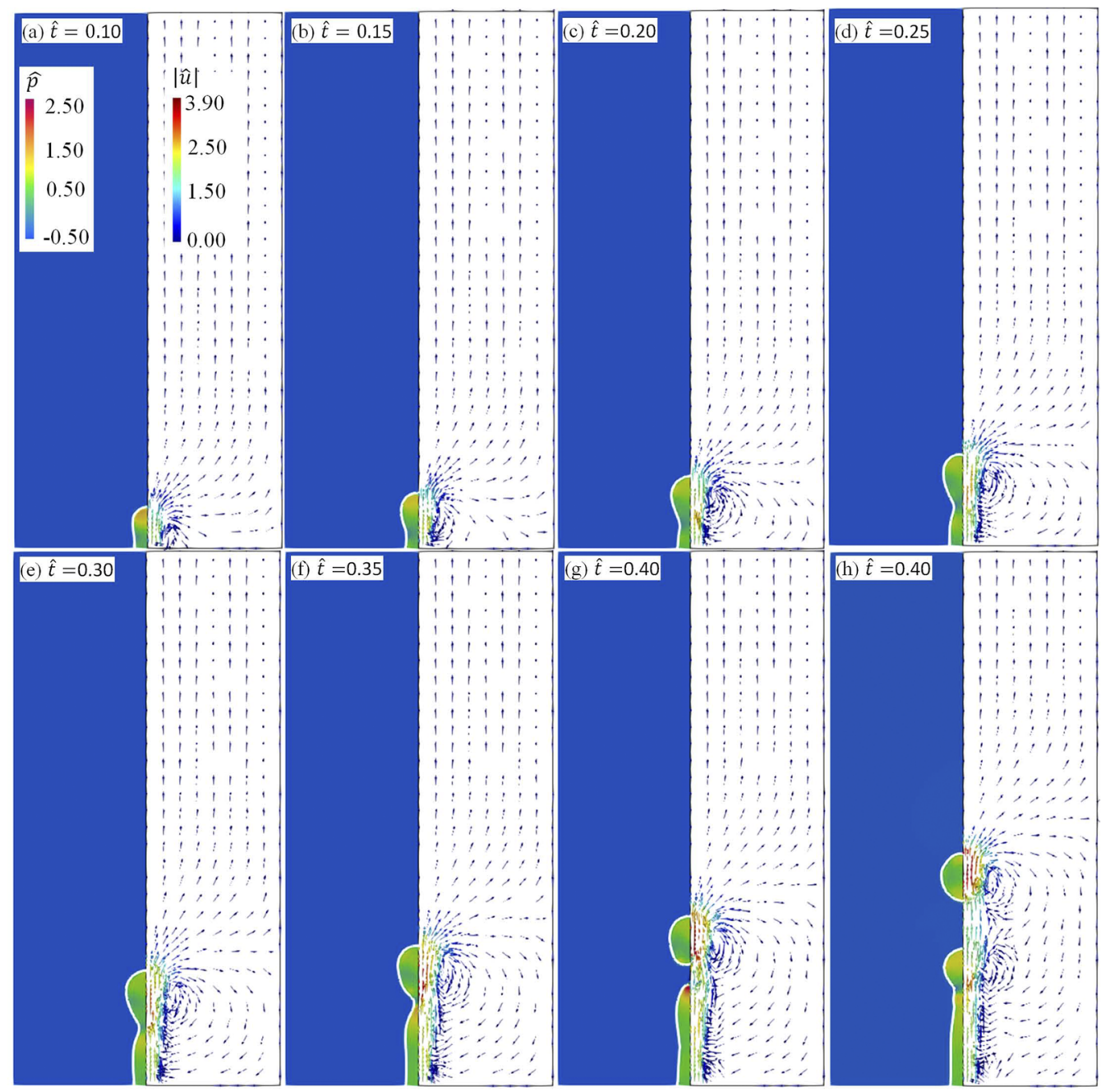

FIG. 15. Temporal evolutions of the jet breakup process under the condition $W e=8.0, B 0=1.4$, and $\tilde{\Omega}=1.0$ during the first half period of acceleration as a comparison to the counterpart in the Faraday-type ligament breakup process shown in Figs. $9(\mathrm{a})-9(\mathrm{~g})$ indicate the time range from 0.10 to 0.40 with a time interval of 0.05 . (h) indicates the simulation results at $\hat{t}=0.5$. The left half shows the pressure contour with white lines denoting the interface. The right half presents the velocity vector colored by its magnitude. 

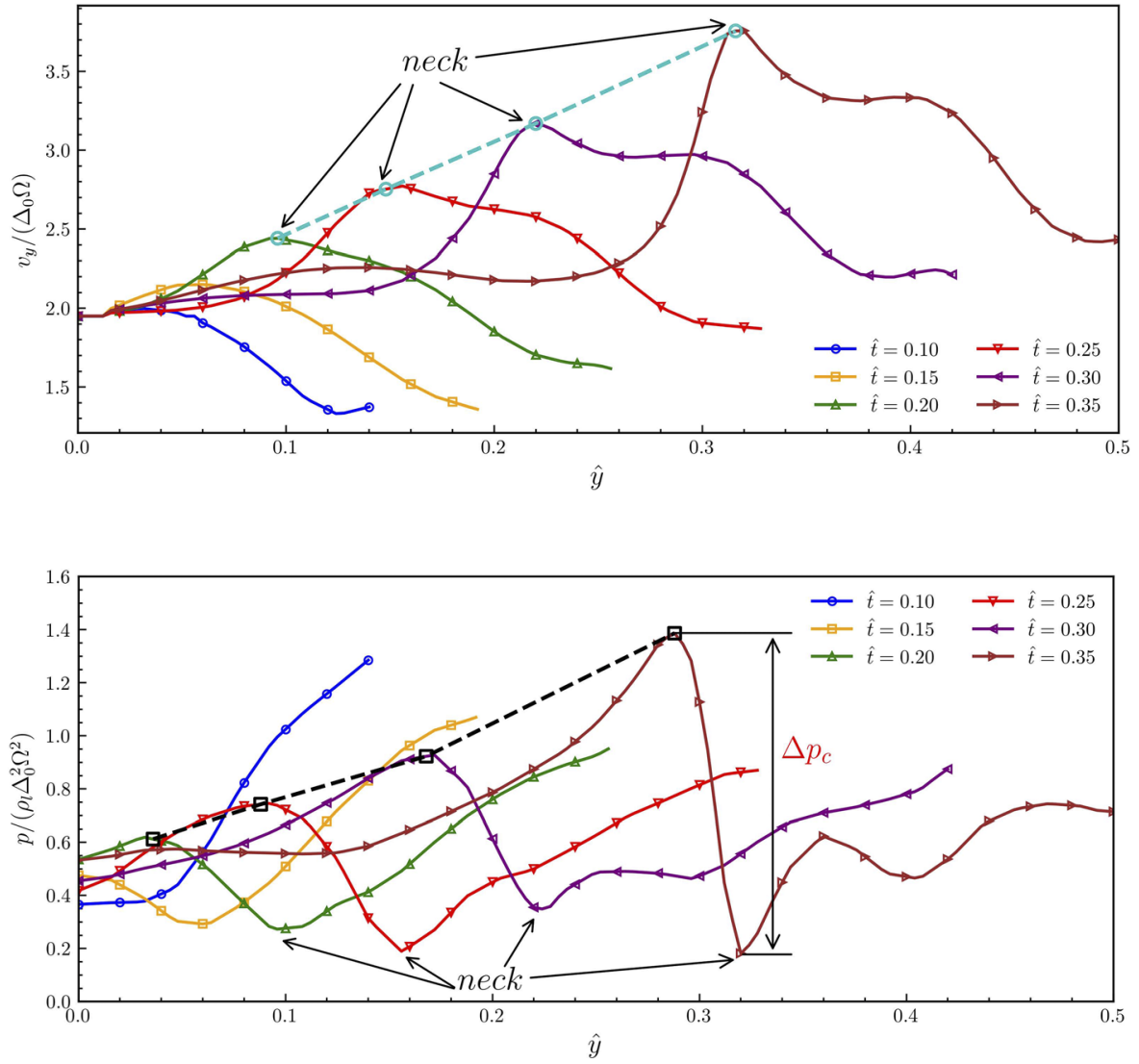

FIG. 16. Distributions of vertical velocities along the jet centerline for the case shown in Fig. 15. The blue dashed line represents the temporal tendency of the peak velocity at the neck.
It shows the temporal evolutions of interface, pressure, and velocity distributions from $\hat{t}=0.1$ to $\hat{t}=0.5$, during which the first breakup occurs. Detailed vertical velocity and pressure distributions along the central line of the jet are depicted in Figs. 16 and 17, respectively. Figure 15 shows that the initially sharp jet tip is immediately rounded by the surface tension force when it issues from orifice. The surface tension force at the jet tip is larger than the external acceleration, which results in the decrease of vertical velocity along downstream of jet before $\hat{t}=0.1$ as shown in Fig. 16. Similar to the breakup process of a Faraday-type ligament, the jet tip contraction causes capillary waves propagating upstream toward the orifice exit. A neck forms upstream the tip bulb after $\hat{t}=0.2$. Figure 17 clearly shows that a pressure difference exists between the neck and its upstream edge, which leads to a fast jet flow in the neck region. As shown in Fig. 16, the velocity of the jet flow maximizes at the neck. This pressure difference increases over time and drives more liquid flowing from the neck to the tip bulb. The neck continues pinching, and breakup occurs at $\hat{t}=0.4$. The newly produced jet tip after the first breakup continues contracting and forms another tip bulb. These dynamics associated with the tip breakup of the liquid jet regime are the same as those in the Faraday-type ligament breakup regime as discussed in Sec. III C (see Figs. 9, 13, and 14 for comparison).

To further quantitatively compare the breakup behaviors between the jet and Faraday-type ligament, many relevant characteristic parameters are compared side-by-side as demonstrated in Table II, including two most important breakup parameters: (1) the capillary wavelength generated by the jet tip contraction reaches a value of $\Lambda / a=3.46$ (3.64 for the corresponding Faradaytype ligament) and (2) the diameter of the disintegrated droplet is $D / a=2.92$ (3.2 for the corresponding Faraday-type ligament). Both the capillary wavelength and the size of the disintegrated droplet of the jet regime are slightly smaller than those of the Faraday-type ligament regime.

Table II also compares the pressure difference between the neck and its upstream edge $\Delta p_{c}$ and the peak velocity at the neck $v_{c}$ just

TABLE II. Comparisons of the capillary wavelength, diameter of the disintegrated droplet, pressure difference between the neck and its upstream edge $\left(\Delta p_{c}\right.$ as shown in Fig. 17), and peak velocity at the neck $\left(v_{c}\right)$ just before breakup between the two breakup regimes.

\begin{tabular}{lcc}
\hline \hline Cases & Faraday-type ligament & Liquid jet \\
\hline $\boldsymbol{\Lambda} / \boldsymbol{a}$ & 3.64 & 3.46 \\
$\boldsymbol{D} / \boldsymbol{a}$ & 3.20 & 2.92 \\
$\Delta \boldsymbol{p}_{\boldsymbol{c}} /\left(\boldsymbol{\rho}_{\boldsymbol{l}} \boldsymbol{\Delta}_{0}^{2} \boldsymbol{\Omega}^{2}\right)$ & 0.83 & 1.18 \\
$\boldsymbol{v}_{\boldsymbol{c}} /\left(\boldsymbol{\Delta}_{0} \boldsymbol{\Omega}\right)$ & 3.12 & 3.74 \\
\hline \hline
\end{tabular}


before the breakup of the tip bulb for the two breakup regimes. It is shown that the pressure difference to drive the jetted flow in the neck region for the jet breakup regime is larger than that for the Faradaytype ligament, which results in a larger peak velocity of the jetted flow at the neck. This would accelerate the pinching process of neck and might inject less liquid volume into the tip bulb through the neck for the jet breakup regime.

Overall, the slight discrepancies between these two breakup regimes are mainly attributed to two effects.

First, the initial conditions for these two breakup regimes are slightly different. For the Faraday-type ligament breakup case, there already exists a column of liquid with surface deformation above the maximum pressure point when it first emerges. Only after this moment, the liquid above the maximum pressure point can be strictly recognized as a ligament that is dynamically freed from the bottom liquid layer. As a result, the upward part of ligament possesses larger velocity at the initial time after which the ligament develops like a jet (see Fig. 7). On the other hand, for the jet breakup case, the initial sharp jet is immediately contracted when it is issued from the orifice. Although the issuing speed is larger than the tip contraction velocity (such that the jet length can grow), compared with the Faraday-type ligament case, the jet length is rather short and the velocity profile is rather flat (except the neck and tip bulb regions) along the $\hat{y}$ direction (as shown in Fig. 16). Consequently, the capillary waves released by the tip contraction can quickly reach the orifice exit and convert into unstable waves after reflection, ${ }^{8}$ which is absent in the Faraday-type ligament breakup process.

Second, in the Faraday-type ligament breakup process, as shown in Fig. 8, the maximum pressure point changes its position over time as well as the vertical velocity there. This corresponds to the orifice moving with a time-dependent issuing speed in the jet breakup regime, which is difficult to be quantitatively identified and numerically reproduced. In the present simulation on the jet breakup regime, we fix the orifice location and use a constant issuing speed, which could result in slightly different developing behaviors from the Faraday-type ligament.

The above preliminary study on the jet breakup under a temporally periodic acceleration and comparison with the Faraday-type ligament breakup indicates that although some small discrepancies exist due to slightly different settings, the two breakup regimes do share the same breakup dynamics at the tip. To bridge the two breakup regimes, the key is to correctly identify the exit condition of a liquid jet corresponding to the time-dependent origin of Faradaytype ligament and quantitatively evaluating the effects of the orifice exit on the jet breakup process. This merits further systematic investigations on the jet breakup with a wide range of controlling parameters.

\section{CONCLUDING REMARKS}

In this paper, we studied ligament formation and breakup caused by Faraday instability using 3D numerical simulation. The numerical scheme used to capture liquid/gas interfacial dynamics was validated with both experimental data and previous simulations. A single-mode Faraday instability with a typical dimensionless parameter combination $(X, Y)$ was simulated as a prototype to elucidate the basic nonlinear dynamics associated with ligament formation and its subsequent breakup.

The simulation indicates that liquid ligament formation is induced by horizontal colliding flow from adjacent trough portions, which enhances the pressure in the ligament root region. The pressure enhancement prevents flow from trough portions and forms a maximum pressure point at the ligament root. This point separates the ligament region from the bulk liquid layer that nearly moved with the bottom substrate as a whole. As a result, the ligament region above the maximum pressure point develops freely and is independent of external vibration.

The ligament tip contracts and generates capillary waves that propagated upstream. The contraction dynamics of the Faradaytype ligament can be well predicted by a one-dimensional theoretical model on a low-speed liquid jet under temporally periodic acceleration. The tip bulb then pinches off due to the short-wave mode instability, and a droplet is generated. The equivalent diameters of disintegrated droplets for the present simulation cases range from $0.32 \lambda$ to $0.375 \lambda$, which are close to the empirical value of $0.34 \lambda$ in Lang's equation.

Finally, we conducted preliminary numerical studies on the evolution of a liquid jet issuing from orifice with constant speed under temporally periodic acceleration to further elucidate the similarity and difference between the Faraday-type ligament breakup and jet breakup regimes. For the jet issue condition corresponding to the present prototype case of Faraday instability, both the capillary wavelength and diameter of the disintegrated droplet are close to each other. It is shown that the two breakup regimes share the same breakup dynamics at the tip (short-wave mode mechanism).

\section{ACKNOWLEDGMENTS}

This work was supported partly by the National Natural Science Foundation of China (Grant No. 51976011) and partly by the LHD Youth Innovation Fund (Grant No. LHD2019CX02). The authors are grateful to the National Supercomputer Center in Tianjin for providing the computational resource. In addition, we acknowledge the invaluable comments of the reviewers, which improved the analyses on the analogy to jet breakup.

\section{APPENDIX A: TIP CONTRACTION VELOCITY}

The ligament tip contraction velocity is derived by considering a 1D model as shown in Fig. 10. The free thread is initially idle, and two tips contract symmetrically due to surface tension. Thus, only half of the thread is focused. Taking the first bulb as the object, for the microgravity condition, the momentum equation

$$
\frac{d}{d t}\left(\rho \pi a^{2} L \frac{d L}{d t}\right)=2 \pi a \sigma-\pi a^{2} \frac{\sigma}{a}
$$

can be built, where $L$ is the distance between the original position of the thread tip and the first neck. Integrating Eq. (A1) over time $t$ yields

$$
L \frac{d L}{d t}=\frac{\sigma}{\rho a} t
$$


which can be further integrated as

$$
\int_{L_{0}}^{L} L d L=\int_{0}^{t} \frac{\sigma}{\rho a} t d t
$$

where $L_{0} \sim O(a)$ is the distance between the tip and the first neck at $t=0$. This produces

$$
L^{2}=L_{0}^{2}+\frac{\sigma}{\rho a} t^{2} .
$$

Combining Eqs. (A2) and (A4) gives an expression for the contraction speed,

$$
U=\frac{d L}{d t}=\frac{\frac{\sigma}{\rho a} t}{\sqrt{L_{0}^{2}+\frac{\sigma}{\rho a} t^{2}}},
$$

which can be approximated as

$$
U=\sqrt{\frac{\sigma}{\rho a}},
$$

when $t \rightarrow \infty$. That is, the contraction velocity $U$ reaches a constant value of $\sqrt{\sigma / \rho a}$ after the initial transition phase of order $\sqrt{\rho a^{3} / \sigma}$.

\section{APPENDIX B: STEADY CAPILLARY WAVELENGTH}

After obtaining the steady contraction velocity $U$, a model is constructed to solve for the steady capillary wavelength caused by contraction. In the coordinate system attached to the moving tip, the fluid from the thread residue enters the bulb with constant velocity $U$. The momentum equation along the axial direction takes the form of

$$
\rho\left(\frac{\partial u}{\partial t}+u \frac{\partial u}{\partial x}\right)=-\frac{\partial p}{\partial x}
$$

with the liquid pressure $p$ satisfying the Laplace condition,

$$
p-p_{g}=\frac{\sigma}{r\left[1+\left(\frac{\partial r}{\partial x}\right)^{2}\right]^{1 / 2}}-\frac{\sigma \frac{\partial^{2} r}{\partial x^{2}}}{\left[1+\left(\frac{\partial r}{\partial x}\right)^{2}\right]^{3 / 2}} .
$$

The $1 \mathrm{D}$ continuity equation can be written based on conservation of mass,

$$
\frac{\partial r}{\partial t}+u \frac{\partial r}{\partial x}=-\frac{r}{2} \frac{\partial u}{\partial x}
$$

By assuming that the gaseous pressure $p_{g}$ is negligibly small compared to the liquid pressure, we substitute Eq. (B2) and $r=a+\delta$, $u=U+u^{\prime}$, where $\delta$ and $u^{\prime}$ are small perturbations, into Eqs. (B1) and (B3) and get the linearized form of

$$
\rho\left(\frac{\partial u^{\prime}}{\partial t}+U \frac{\partial u^{\prime}}{\partial x}\right)=\sigma \frac{\partial}{\partial x}\left[\frac{\delta}{a^{2}}+\frac{\partial^{2} \delta}{\partial x^{2}}\right]
$$

and

$$
\frac{\partial \delta}{\partial t}+U \frac{\partial \delta}{\partial x}=-\frac{a}{2} \frac{\partial u^{\prime}}{\partial x}
$$

upon neglecting small values of order 2 and higher. Since we are interested only in the steady solution, the unsteady terms in Eqs. (B4) and (B5) can be dropped to produce

$$
\rho U \frac{d u^{\prime}}{d x}=\sigma \frac{d}{d x}\left[\frac{\delta}{a^{2}}+\frac{d^{2} \delta}{d x^{2}}\right]
$$

and

$$
U \frac{d \delta}{d x}=-\frac{a}{2} \frac{d u^{\prime}}{d x} .
$$

Substituting Eq. (B6) into Eq. (B7) leads to

$$
U \frac{d \delta}{d x}=-\frac{a}{2} \frac{1}{\rho U} \sigma \frac{d}{d x}\left[\frac{\delta}{a^{2}}+\frac{d^{2} \delta}{d x^{2}}\right],
$$

which is further reduced to

$$
\frac{d^{2} \delta}{d x^{2}}+\left[\frac{1}{a^{2}}+\frac{2 \rho U^{2}}{a \sigma}\right] \delta=0
$$

after integrating Eq. (B8) over $x$.

Clearly, the solution of Eq. (B9) is a simple harmonic wave with wavenumber $k=\sqrt{\frac{1}{a^{2}}+\frac{2 \rho U^{2}}{a \sigma}}$. This indicates that the wavelength of a steady capillary wave on a thread or ligament is

$$
\Lambda=\frac{2 \pi}{k}=\frac{2 \pi}{\sqrt{\frac{1}{a^{2}}+\frac{2 \rho U^{2}}{a \sigma}}}=\frac{2 \pi}{\sqrt{3}} a \approx 3.63 a
$$

if Eq. (A6) is applied.

\section{APPENDIX C: TIP CONTRACTION VELOCITY FOR ACCELERATING JET}

For a liquid jet issuing from a nozzle with a velocity $V$ and developing under a temporally periodic acceleration of $A_{0} \sin (\Omega t)$, we can build the momentum equation for the first bulb as

$$
\frac{d}{d t}\left(\rho \pi a^{2} L \frac{d L}{d t}\right)=2 \pi a \sigma-\pi a^{2} \frac{\sigma}{a}-\rho \pi a^{2} L A_{0} \sin (\Omega t),
$$

which is reduced to

$$
L \frac{d^{2} L}{d t^{2}}+\left(\frac{d L}{d t}\right)^{2}=\frac{\sigma}{\rho a}-A_{0} \sin (\Omega t) L .
$$

Taking $s=d L / d t$ produces

$$
\frac{2}{L} s^{2}+\frac{d s^{2}}{d L}=\frac{2 \sigma}{\rho a L}-2 A_{0} \sin (\Omega t)
$$

or

$$
\frac{d\left(L^{2} s^{2}\right)}{d L}=\frac{2 \sigma}{\rho a} L-2 A_{0} \sin (\Omega t) L^{2} .
$$

Integrating Eq. (C4) from $L_{0}$ to $L$ produces

$$
L^{2} s^{2}=\frac{\sigma}{\rho a}\left(L^{2}-L_{0}^{2}\right)-\frac{2}{3} A_{0} \sin (\Omega t)\left(L^{3}-L_{0}^{3}\right) .
$$

Solving Eq. (C5) gives

$$
s=\frac{d L}{d t}= \pm \sqrt{\frac{\sigma}{\rho a}-\frac{2}{3} A_{0} \sin (\Omega t) L-\frac{L_{0}^{2}\left(\frac{\sigma}{\rho a}-\frac{2}{3} A_{0} \sin (\Omega t) L_{0}\right)}{L^{2}}},
$$

which takes the dimensionless form as

$$
\frac{d \tilde{L}}{d \tilde{t}}= \pm \sqrt{1-\frac{2}{3} \gamma_{0} \sin (\tilde{\Omega} \tilde{t}) \tilde{L}-\frac{\left(1-\frac{2}{3} \gamma_{0} \sin (\tilde{\Omega} \tilde{t})\right)}{\tilde{L}^{2}}}
$$


with the dimensionless quantities $\tilde{L}=L / L_{0}, \tilde{\Omega}=\Omega L_{0} / \sqrt{\sigma / \rho a}$, $\tilde{t}=t \sqrt{\sigma / \rho a} / L_{0}$, and $\gamma(\tilde{t})=\gamma_{0} \sin (\tilde{\Omega} \tilde{t})=\rho a L_{0} A_{0} \sin (\tilde{\Omega} \tilde{t}) / \sigma$.

\section{DATA AVAILABILITY}

Data that support the findings of this study are available from the corresponding author upon reasonable request.

\section{REFERENCES}

${ }^{1}$ A. H. Lefebvre and V. G. McDonell, Atomization and Sprays (CRC Press, 2017).

${ }^{2}$ S. P. Lin and R. D. Reitz, "Drop and spray formation from a liquid jet," Annu. Rev. Fluid Mech. 30, 85-105 (1998).

${ }^{3}$ W. Jones, A. Marquis, and K. Vogiatzaki, "Large-eddy simulation of spray combustion in a gas turbine combustor," Combust. Flame 161, 222-239 (2014).

${ }^{4} \mathrm{~W}$. Yang, M. Jia, K. Sun, and T. Wang, "Influence of density ratio on the secondary atomization of liquid droplets under highly unstable conditions," Fuel 174, $25-35$ (2016).

${ }^{5}$ J. S. Shrimpton, "Atomization, combustion, and control of charged hydrocarbon sprays,” Atomization Sprays 11, 365-396 (2001).

${ }^{6}$ J. Eggers and E. Villermaux, "Physics of liquid jets," Rep. Prog. Phys. 71, 036601 (2008).

${ }^{7}$ J. Shinjo and A. Umemura, "Simulation of liquid jet primary breakup: Dynamics of ligament and droplet formation," Int. J. Multiphase Flow 36, 513-532 (2010).

${ }^{8}$ A. Umemura, "Self-destabilizing mechanism of a laminar inviscid liquid jet issuing from a circular nozzle," Phys. Rev. E 83, 046307 (2011).

${ }^{9}$ Y. Fan, N. Hashimoto, H. Nishida, and Y. Ozawa, "Spray characterization of an air-assist pressure-swirl atomizer injecting high-viscosity Jatropha oils," Fuel 121, 271-283 (2014).

${ }^{10}$ S. Huang, P. Deng, R. Huang, Z. Wang, Y. Ma, and H. Dai, "Visualization research on spray atomization, evaporation and combustion processes of ethanoldiesel blend under LTC conditions," Energy Convers. Manage. 106, 911-920 (2015).

${ }^{11}$ H. K. Suh and C. S. Lee, "A review on atomization and exhaust emissions of a biodiesel-fueled compression ignition engine," Renewable Sustainable Energy Rev. 58, 1601-1620 (2016)

${ }^{12}$ M. K. Tan, J. R. Friend, O. K. Matar, and L. Y. Yeo, "Capillary wave motion excited by high frequency surface acoustic waves," Phys. Fluids 22, 112112 (2010).

${ }^{13} \mathrm{M}$. K. Tan, J. R. Friend, and L. Y. Yeo, "Interfacial jetting phenomena induced by focused surface vibrations," Phys. Rev. Lett. 103, 024501 (2009).

${ }^{14} \mathrm{~J}$. Wang, H. Hu, A. Ye, J. Chen, and P. Zhang, "Experimental investigation of surface acoustic wave atomization," Sens. Actuators, A 238, 1-7 (2016).

${ }^{15}$ B. A. Puthenveettil and E. Hopfinger, "Evolution and breaking of parametrically forced capillary waves in a circular cylinder," J. Fluid Mech. 633, 355 (2009).

${ }^{16}$ B. Vukasinovic, M. K. Smith, and A. Glezer, "Spray characterization during vibration-induced drop atomization," Phys. Fluids 16, 306-316 (2004).

${ }^{17}$ F. Liu, N. Kang, Y. Li, and Q. Wu, "Experimental investigation on the atomization of a spherical droplet induced by Faraday instability," Exp. Therm. Fluid Sci. 100, 311-318 (2019).

${ }^{18}$ C. Goodridge, H. Hentschel, and D. Lathrop, "Breaking Faraday waves: Critical slowing of droplet ejection rates," Phys. Rev. Lett. 82, 3062-3065 (1999).

${ }^{19}$ C. L. Goodridge, W. T. Shi, H. Hentschel, and D. P. Lathrop, "Viscous effects in droplet-ejecting capillary waves," Phys. Rev. E 56, 472-475 (1997).

${ }^{20}$ A. James, B. Vukasinovic, M. K. Smith, and A. Glezer, "Vibration-induced drop atomization and bursting," J. Fluid Mech. 476, 1-28 (2003).

${ }^{21}$ A. J. James, M. K. Smith, and A. Glezer, "Vibration-induced drop atomization and the numerical simulation of low-frequency single-droplet ejection," J. Fluid Mech. 476, 29-62 (2003).

${ }^{22}$ S. C. Tsai, S. K. Lin, R. W. Mao, and C. S. Tsai, "Ejection of uniform micrometersized droplets from Faraday waves on a millimeter-sized water drop," Phys. Rev. Lett. 108, 154501 (2012).
${ }^{23}$ J. C. Simon, O. A. Sapozhnikov, V. A. Khokhlova, L. A. Crum, and M. R. Bailey, "Ultrasonic atomization of liquids in drop-chain acoustic fountains," J. Fluid Mech. 766, 129-146 (2015).

${ }^{24}$ R. J. Lang, "Ultrasonic atomization of liquids," J. Acoust. Soc. Am. 34, 6-8 (1962).

${ }^{25}$ F. Barreras, H. Amaveda, and A. Lozano, "Transient high-frequency ultrasonic water atomization," Exp. Fluids 33, 405-413 (2002).

${ }^{26}$ N. L. Mai, Y.-M. Koo, and S. H. Ha, "Separation characteristics of hydrophilic ionic liquids from ionic liquids-water solution by ultrasonic atomization," Ultrason. Sonochem. 53, 187-191 (2019).

${ }^{27}$ P. K. Notz and O. A. Basaran, "Dynamics and breakup of a contracting liquid filament," J. Fluid Mech. 512, 223-256 (2004).

${ }^{28}$ D. W. Bousfield, I. H. Stockel, and C. K. Nanivadekar, "The breakup of viscous jets with large velocity modulations," J. Fluid Mech. 218, 601-617 (2006).

${ }^{29}$ H. Ding, E. Q. Li, F. H. Zhang, Y. Sui, P. D. M. Spelt, and S. T. Thoroddsen, "Propagation of capillary waves and ejection of small droplets in rapid droplet spreading," J. Fluid Mech. 697, 92-114 (2012).

${ }^{30}$ A. A. Castrejón-Pita, J. R. Castrejón-Pita, and I. M. Hutchings, "Breakup of liquid filaments," Phys. Rev. Lett. 108, 074506 (2012).

${ }^{31}$ D. R. Webster and E. K. Longmire, "Jet pinch-off and drop formation in immiscible liquid-liquid systems," Exp. Fluids 30, 47-56 (2001).

${ }^{32}$ C. Xu, Z. Zhang, J. Fu, and Y. Huang, "Study of pinch-off locations during dropon-demand inkjet printing of viscoelastic alginate solutions," Langmuir 33, 50375045 (2017).

${ }^{33}$ S. H. Sadek, H. H. Najafabadi, and F. J. Galindo-Rosales, "Capillary breakup extensional magnetorheometry," J. Rheol. 64, 55-65 (2019).

${ }^{34}$ S. Santra, S. Das, and S. Chakraborty, "Electric field-induced pinch-off of a compound droplet in Poiseuille flow," Phys. Fluids 31, 062004 (2019).

${ }^{35} \mathrm{R}$. Reitz, "Modeling atomization processes in high-pressure vaporizing sprays," Atomization Spray Technol. 3, 309-337 (1987).

${ }^{36}$ T. Su, M. Patterson, R. D. Reitz, and P. Farrell, "Experimental and numerical studies of high pressure multiple injection sprays," in SAE Transactions (SAE International, 1996), Vol. 105, pp. 1281-1292.

${ }^{37}$ P. J. O'Rourke and A. A. Amsden, “The TAB method for numerical calculation of spray droplet breakup," SAE Technical Paper No. 872089, 1987.

${ }^{38}$ M. Faraday, "XVII. On a peculiar class of acoustical figures; and on certain forms assumed by groups of particles upon vibrating elastic surfaces," Philos. Trans. R. Soc. London 121, 299-340 (1831).

${ }^{39}$ A. Umemura and J. Shinjo, "Detailed SGS atomization model and its implementation to two-phase flow LES," Combust. Flame 195, 232-252 (2018).

${ }^{40}$ A. H. E. Adou and L. S. Tuckerman, "Faraday instability on a sphere: Floquet analysis," J. Fluid Mech. 805, 591-610 (2016).

${ }^{41}$ T. B. Benjamin and F. Ursell, "The stability of the plane free surface of a liquid in vertical periodic motion," Proc. R. Soc. London, Ser. A 225, 505-515 (1954).

${ }^{42}$ Y. Li, P. Zhang, and N. Kang, "Linear analysis on the interfacial instability of a spherical liquid droplet subject to a radial vibration," Phys. Fluids 30, 102104 (2018).

${ }^{43}$ P. Chen and J. Viñals, "Pattern selection in Faraday waves," Phys. Rev. Lett. 79, 2670 (1997).

${ }^{44}$ M.-T. Westra, D. J. Binks, and W. Van De Water, "Patterns of Faraday waves," J. Fluid Mech. 496, 1-32 (2003).

${ }^{45} \mathrm{~W}$. Zhang and J. Viñals, "Pattern formation in weakly damped parametric surface waves," J. Fluid Mech. 336, 301-330 (1997).

${ }^{46} \mathrm{Y}$. Li and A. Umemura, "Threshold condition for spray formation by Faraday instability,” J. Fluid Mech. 759, 73-103 (2014).

${ }^{47} \mathrm{Y}$. Li and A. Umemura, "Two-dimensional numerical investigation on the dynamics of ligament formation by Faraday instability," Int. J. Multiphase Flow 60, 64-75 (2014).

${ }^{48}$ Y. Renardy and M. Renardy, "PROST: A parabolic reconstruction of surface tension for the volume-of-fluid method," J. Comput. Phys. 183, 400-421 (2002).

${ }^{49}$ M. M. Francois, S. J. Cummins, E. D. Dendy, D. B. Kothe, J. M. Sicilian, and M. W. Williams, "A balanced-force algorithm for continuous and sharp interfacial surface tension models within a volume tracking framework," J. Comput. Phys. 213, 141-173 (2006). 
${ }^{50}$ S. J. Cummins, M. M. Francois, and D. B. Kothe, "Estimating curvature from volume fractions," Comput. Struct. 83, 425-434 (2005).

${ }^{51}$ M. Rudman, "Volume-tracking methods for interfacial flow calculations," Int. J. Numer. Methods Fluids 24, 671-691 (1997).

${ }^{52}$ S. Popinet, "An accurate adaptive solver for surface-tension-driven interfacial flows,” J. Comput. Phys. 228, 5838-5866 (2009).

${ }^{53} \mathrm{~A}$. J. Chorin, "On the convergence of discrete approximations to the NavierStokes equations," Math. Comput. 23, 341-353 (1969).

${ }^{54}$ S. Popinet, "Gerris: A tree-based adaptive solver for the incompressible Euler equations in complex geometries," J. Comput. Phys. 190, 572-600 (2003).

${ }^{55}$ L. Jiang, C.-L. Ting, M. Perlin, and W. W. Schultz, "Moderate and steep Faraday waves: Instabilities, modulation and temporal asymmetries," J. Fluid Mech. 329, 275-307 (1996).
${ }^{56}$ J. Wright, S. Yon, and C. Pozrikidis, "Numerical studies of two-dimensional Faraday oscillations of inviscid fluids," J. Fluid Mech. 402, 1-32 (2000).

${ }^{57}$ A. Umemura, "Self-destabilizing mechanism of circular liquid jet first report: Capillary waves associated with liquid jet destabilization," J. Jpn. Soc. Aeronaut. Space Sci. 55, 216-223 (2007).

${ }^{58}$ A. Umemura, S. Kawanabe, H. Kojika, F. Chen, and J. Shinjo, "Findings from microgravity experiments on low-speed water jet disintegration," J. Jpn. Soc. Aeronaut. Space Sci. 58, 15-23 (2010).

${ }^{59}$ A. Umemura, S. Kawanabe, S. Suzuki, and J. Osaka, "Two-valued breakup length of a water jet issuing from a finite-length nozzle under normal gravity," Phys. Rev. E 84, 036309 (2011).

${ }^{60}$ A. Umemura, "A theoretical study on short-wavelength disintegration mechanism of liquid ligaments,” J. Jpn. Soc. Aeronaut. Space Sci. 56, 228-238 (2008). 